\title{
Fluctuation of 400-Hz Sound Intensity in the 2001 ASIAEX South China Sea Experiment
}

\author{
Timothy F. Duda, James F. Lynch, Senior Member, IEEE, Arthur E. Newhall, Lixin Wu, and Ching-Sang Chiu
}

\begin{abstract}
We present analyses of fluctuations seen in acoustic signals transmitted by two $400-\mathrm{Hz}$ sources moored as part of the ASIAEX 2001 South China Sea (SCS) experiment. One source was near the bottom in $350-\mathrm{m}$ deep water $31.3 \mathrm{~km}$ offshore from the receiving array, and the other was near the bottom in $135-\mathrm{m}$ deep water $20.6 \mathrm{~km}$ alongshore from the array. Time series of signal intensity measured at individual phones of a 16-element vertical line array are analyzed, as well as time series of intensity averaged over the array. Signals were recorded from 2 May to 17 May 2001. Fluctuations were observed at periods ranging from subtidal (days) to the shortest periods resolved with our signaling (10 s). Short-period fluctuations of depth- and time-averaged intensity have scintillation indexes (computed within 3-h long windows) which peak at values near 0.5 during an interval of numerous high-amplitude internal gravity waves, and which are lower during intervals with fewer internal waves. The decorrelation times of the averaged intensity (energy level) are also closely related to internal wave properties. Scintillation indexes computed for unaveraged pulses arriving at individual phones often exceed unity.
\end{abstract}

Index Terms-Acoustic intensity fluctuation, underwater acoustic propagation.

\section{INTRODUCTION}

A NUMBER of moored sound sources were deployed during the Asian Seas International Acoustics Experiment (ASIAEX) South China Sea (SCS) acoustic propagation study, sending a variety of phase, amplitude, and frequency modulated signals to a centrally placed, moored horizontal and vertical line array receiver (HLA/VLA). The geometry is shown in Fig. 1. In this paper, we present characteristics of broadband pulses transmitted from two $400 \mathrm{~Hz}$ sources deployed in that experiment, one placed down-slope from the receiver and one placed roughly along-slope. In particular, we will examine the travel time fluctuations and (mainly) the amplitude/intensity fluctuations of the pulses. This paper is similar in spirit to that of the recent SWARM and PRIMER experiments in the Mid-Atlantic Bight/Southern New England area [1]-[3]. Note that the characteristics of acoustic transmissions at other frequencies are discussed in other papers in this volume [4], [5].

The ASIAEX experiment had a few technical improvements over the previous experiments. 1) A longer time series was ob-

Manuscript received May 7, 2003; revised April 7, 2004. This work was supported by the U.S. Office of Naval Research.

T. F. Duda, J. F. Lynch, and A. E. Newhall are with the Applied Ocean Physics and Engineering Department, Woods Hole Oceanographic Institution, Woods Hole, MA 02543 USA (e-mail: tduda@whoi.edu; jlynch@whoi.edu).

L. Wu is with the National Laboratory of Acoustics, Chinese Academy of Sciences, Beijing, People's Republic of China.

C.-S. Chiu is with the Department of Oceanography, Naval Postgraduate School, Monterey, CA 93943 USA.

Digital Object Identifier 10.1109/JOE.2004.836997 tained, with over 2 wk of simultaneous acoustics and oceanography measurements (i.e., a full spring-neap tidal cycle). 2) Both along-shelf and across-shelf geometries were explored. 3) There was a higher degree of oceanographic and geologic environmental support for the acoustics measurements. 4) Both horizontal and vertical array receptions were available, providing measurements of azimuthal as well as vertical angle dependencies of the acoustic field.

This paper is organized as follows. In Section II, we discuss the experimental setup and the area, including the subsurface geology. In Section III, we examine the details of the highly variable physical oceanography, which is the primary driver of the observed acoustic fluctuations. In Section IV, we present the fluctuations and their correlations to the oceanography. Finally, in Section V, we present our conclusion.

\section{EXPERIMENTAL GEOMETRY AND SCHEDULE}

Fig. 1 shows the bathymetry of the study area and the positions of the moored sources, the environmental sensors, and the receiver. Two source clusters were placed along-shelf and across-shelf from the receiver, so that we could examine the differences imposed by the transmission geometry on the propagation. The lengths of the $400-\mathrm{Hz}$ paths were $20.62 \mathrm{~km}$ alongshelf (east of the receiver) and $31.28 \mathrm{~km}$ across-shelf (south of the receiver), not extremely long distances compared to similar experiments and to detection limits, but long enough to display the scattering effects we wish to examine. The sources, at coordinates $21^{\circ} 56.35^{\prime} \mathrm{N}, 117^{\circ} 22.437^{\prime} \mathrm{E}$ (east source), and $21^{\circ} 36.87^{\prime} \mathrm{N} 117^{\circ} 17.229^{\prime} \mathrm{E}$ (south source), were moored $13 \mathrm{~m}$ above the bottom (at instrument depths of 100 and $330 \mathrm{~m}$, respectively) to exploit the near bottom acoustic duct and to reduce mooring motion effects. A 300- and a 500-Hz source were also moored at the eastern site and a $224-\mathrm{Hz}$ source was moored at the southern site. Pulse transmissions were recorded for a period of greater than $2 \mathrm{wk}$ (2 May to 17 May 2001), enabling measurement of the variability of the acoustic and oceanographic fields over a full spring-neap tidal cycle. At $400 \mathrm{~Hz}$, bursts of 88 coded pulses having $5.11 \mathrm{~s}$ duration were sent every $30 \mathrm{~min}$ for the first $9.5 \mathrm{~d}$ and 23 pulse bursts were sent every $10 \mathrm{~min}$ for the final $5.7 \mathrm{~d}$. Further information is contained in a report [6].

The WHOI/NPS acoustic receiver array resided at $21^{\circ} 52.82^{\prime} \mathrm{N}, 117^{\circ} 11.08^{\prime} \mathrm{E}$ in $124-\mathrm{m}$ water depth. In this paper, we will concentrate on the vertical line array (VLA) hydrophone receptions from this system. We will also present data from Loco Mooring 1 at $21^{\circ} 55.71^{\prime} \mathrm{N}, 117^{\circ} 9.18^{\prime} \mathrm{E}$ at $109-\mathrm{m}$ depth, a near-receiver environmental mooring at $21^{\circ} 53.94^{\prime} \mathrm{N}$, $117^{\circ} 10.62^{\prime} \mathrm{E}$ at 120 -m depth, a mooring near the southern source at $21^{\circ} 36.87^{\prime} \mathrm{N}, 117^{\circ} 16.98^{\prime} \mathrm{E}$ at $350-\mathrm{m}$ depth, and one 


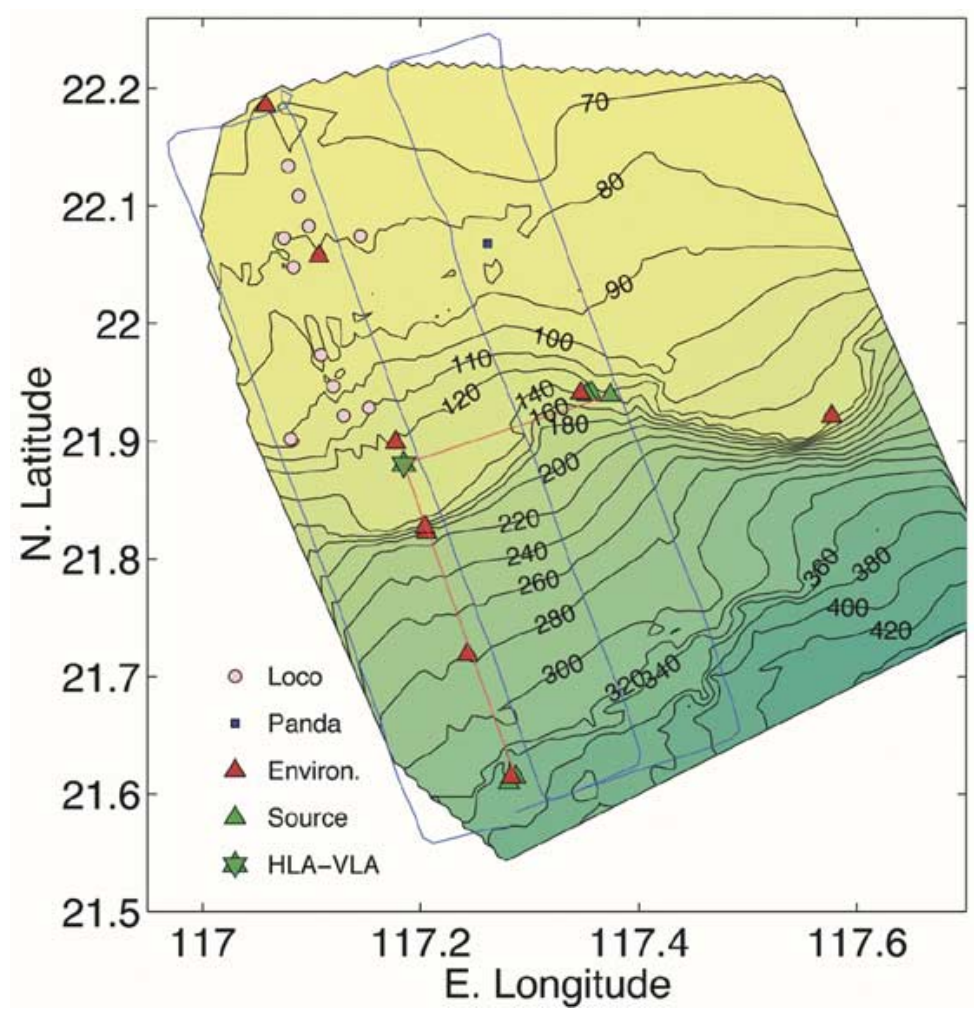

Fig. 1. Plan view of the bathymetry and the deployment pattern of recovered acoustic and oceanographic instruments. Other equipment was lost. Red lines show the two $400-\mathrm{Hz}$ propagation paths to the horizontal line array/vertical line array receiver. PANDA is a single-hydrophone acoustic receiver. LOCOs are temperature-pressure moorings. Environmental moorings measured currents, temperature, pressure, and conductivity.

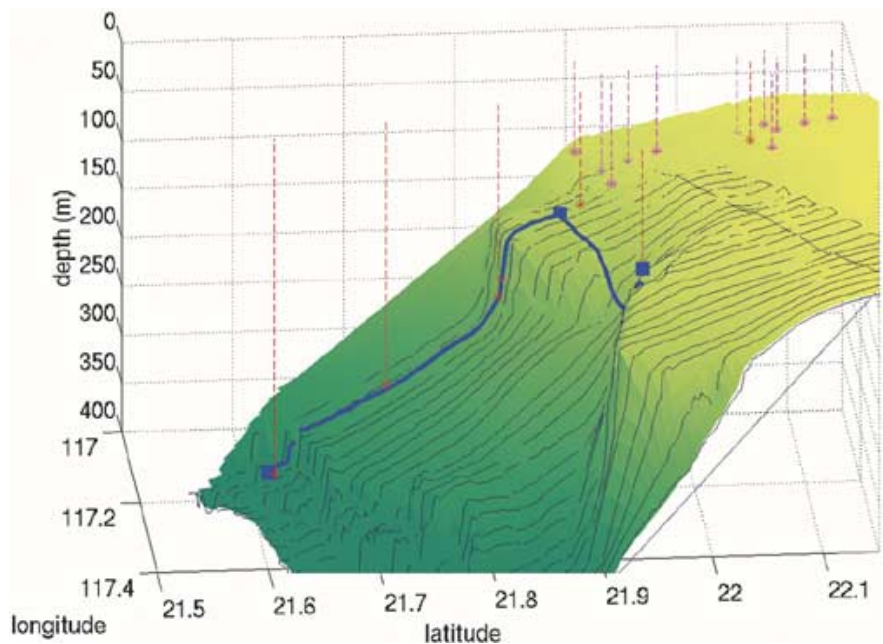

Fig. 2. A 3-D view of the bathymetry of the area, and of the oceanographic and acoustic moorings deployed. The projections of the 400-Hz acoustic paths on the bottom are depicted as blue lines.

at $21^{\circ} 49.35^{\prime} \mathrm{N}, 117^{\circ} 12.33^{\prime} \mathrm{E}$ at $202-\mathrm{m}$ depth. The final three listed moorings were on the southern transmission path.

The bathymetry of the area is shown in Fig. 2, along with a three-dimensional (3-D) representation of the moored instruments. It is seen that both the along-shelf (eastern) and acrossshelf (southern) paths contain some highly irregular bathymetry, which is typical of this region. Geoacoustically, the chirp sonar survey by Schock [7] shows that the surficial sediments are composed of sand at the source and receiver locations, with patches of sandy-silt in between. This survey provides a first-order geoa- coustic model which is consistent with other data [8], and can be used to improve acoustic propagation modeling for the area.

\section{PhysicAl OCEANOGRAPHIC ENVIRONMENT}

We next consider the physical oceanographic environmental factors that were largely responsible for the observed fluctuations. The experimental period in spring 2001 displayed only a small amount of mesoscale variability [9], so we can neglect the shipboard measurements and concentrate on the moored mea- 


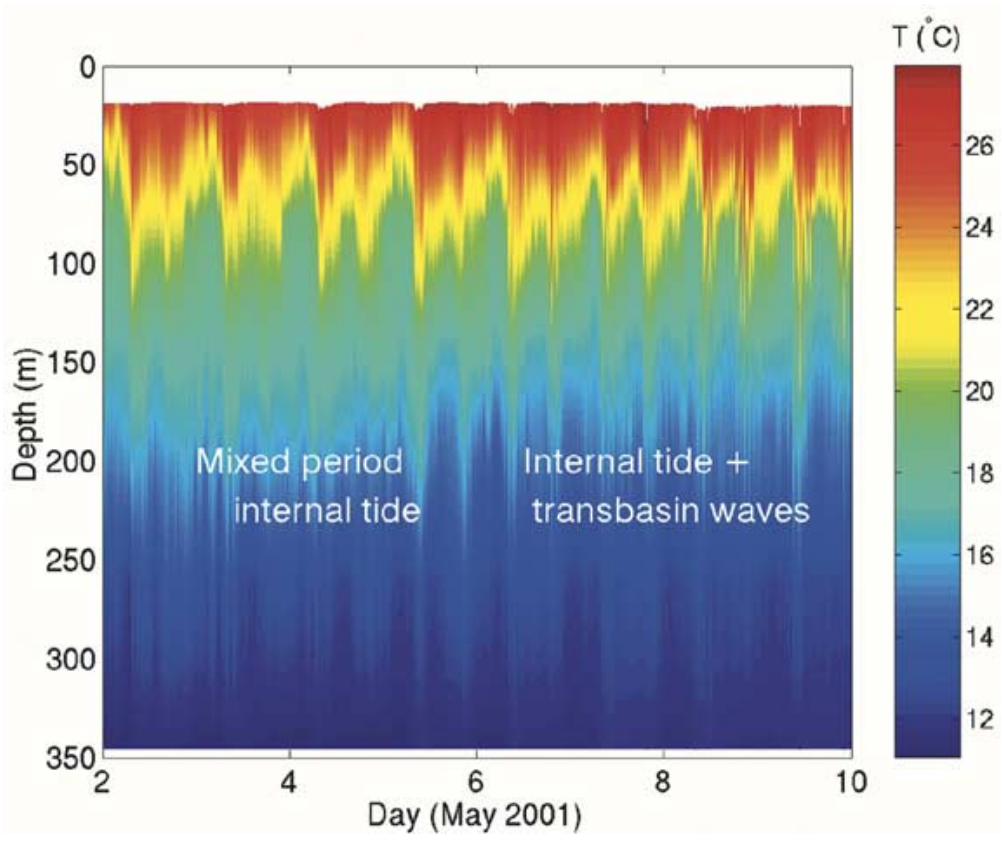

Fig. 3. Portion of the temperature time series at the 350-m mooring, showing the strong mixed (diurnal and semidiurnal) internal tides, as well as the very large "transbasin" solitary waves, which originate in the Luzon Strait. Temperature measured at nine levels once per minute is shown, with interpolation between the levels.

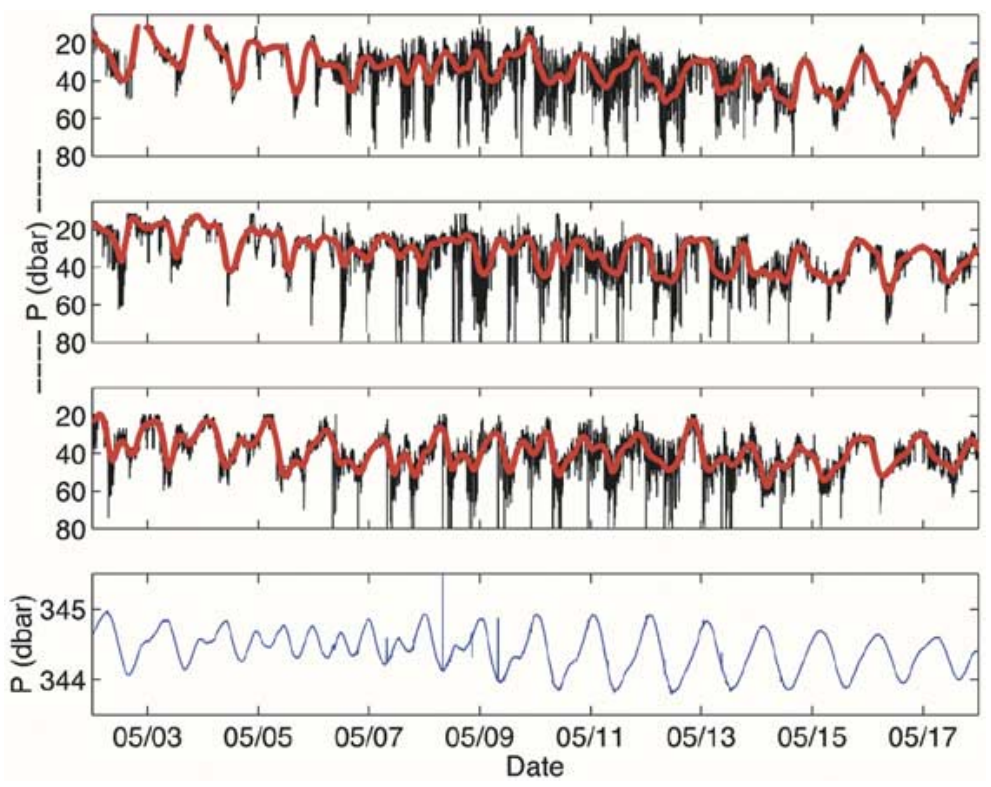

Fig. 4. The top three panels illustrate the internal wave activity during the experimental period via the $25^{\circ} \mathrm{C}$ isotherm height at the (from top to bottom) $120-, 200-$, and 350-m depth across-shelf mooring sites. The lower panel shows the bottom pressure record at the 350-m site.

surements which clearly show the internal-wave band oceanography. The temperature signatures of the internal tide and internal solitary-type wave activity at the 350 -m oceanographic mooring (located on the across-shelf acoustic path) are shown in Fig. 3. Mixed diurnal and semidiurnal tidal-band thermocline displacements (internal tides) are visible, as well as high-frequency nonlinear (solitary-type) waves. Note that although the internal-wave induced temperature perturbation extends down to appreciable depths, the near-bottom temperature (thus, sound speed) disturbance in the water is comparatively small. Therefore, the scattering of low-order acoustic modes, which have turning points closer to the bottom because the profile is downward refracting, may be weaker than that of high-order modes.

The top three panels of Fig. 4 illustrate the internal wave activity during the experimental period by showing the $25^{\circ} \mathrm{C}$ isotherm displacement $\eta$ at the 120-, 200-, and 350-m isobath across-shelf mooring sites. The black lines show the entire time series of $\eta$, while the red lines show filtered $\eta$ in the tidal band. These panels show an important aspect of the internal wave field at the site; namely, three distinct periods of internal wave activity. In the first period, from 2 to 6 May, one sees a strong diurnal internal tide component, but little solitary wave activity. 


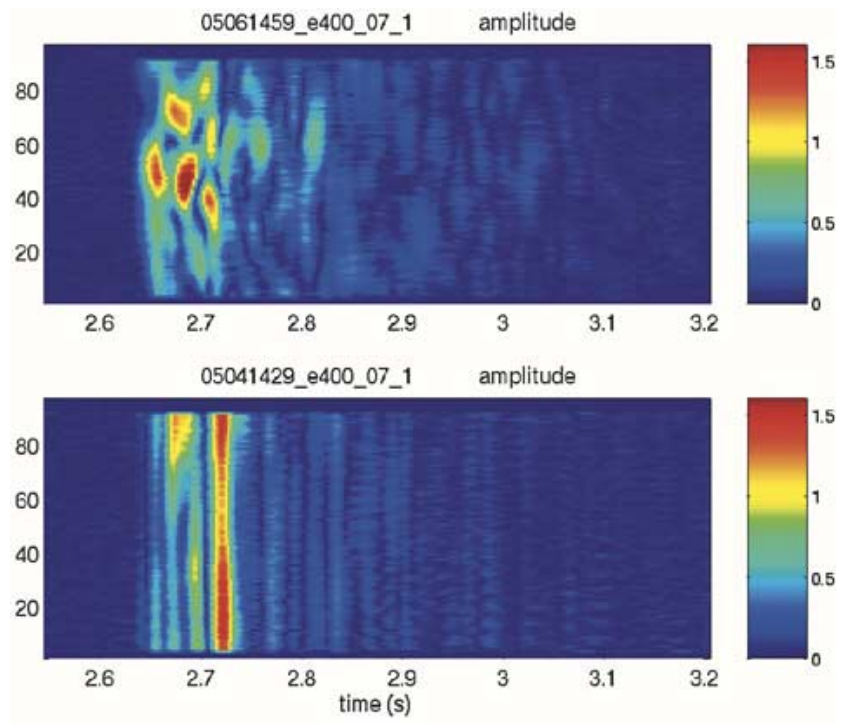

Fig. 5. Two series of receptions at the WHOI/NPS VLA at two different days, 6 May (top panel) and 4 May (bottom panel) showing clear differences in structure. The vertical coordinate is the index number of the pulse reception. The pulse repetition interval is $5.11 \mathrm{~s}$. Amplitude of matched-filter output is indicated by color.

From 6 to 14 May, the semidiurnal internal tidal component is more pronounced and there is a substantial high-frequency internal wave field. After 14 May (until the 18th), the conditions revert to those of the first period. In the bottom panel of Fig. 4, we show bottom pressure measurements of barotropic surface tidal elevation at the 350-m site, which has roughly 1 -m excursion (peak to peak), is mixed/diurnal dominant and is one of the important factors determining the internal wave field.

\section{ACOUSTIC VARIABILITY AND ITS Relation TO OCEANOGRAPHY}

Due to their strong correlation with the oceanography, the acoustics show subtidal, tidal, and high-frequency variability. (We refer to ocean frequency here, the acoustic frequency is fixed at $400 \mathrm{~Hz}$.) In this paper, we will concentrate on long-period modulation of high-frequency acoustic variability, caused directly by fortnightly (or similar) tidal control of nonlinear (steep) internal waves. Quantities we will investigate are

1) intensity "point statistics" (in space and time);

2) time series of pulse-duration averaged intensity $E_{t}$;

3) depth averaged $E_{t}$ values, denoted $E_{t z}$ (both $E_{t}$ and $E_{t z}$ are incoherent sums);

4) correlation time scale of $E_{t z}$;

5) highest peak intensities within pulses at single phones.

These are defined in Section IV-B. They are later compared with the tidally modulated statistics of the high-frequency internal waves.

\section{A. Acoustic Reception Characteristics}

Before proceeding into the data analyses, we should point out that the vertical extent of the WHOI/NPS VLA/HLA was from $121 \mathrm{~m}$ ( $3 \mathrm{~m}$ above the bottom) to $42 \mathrm{~m}$, which was not the full extent of the water column. This was due to our moving the array to

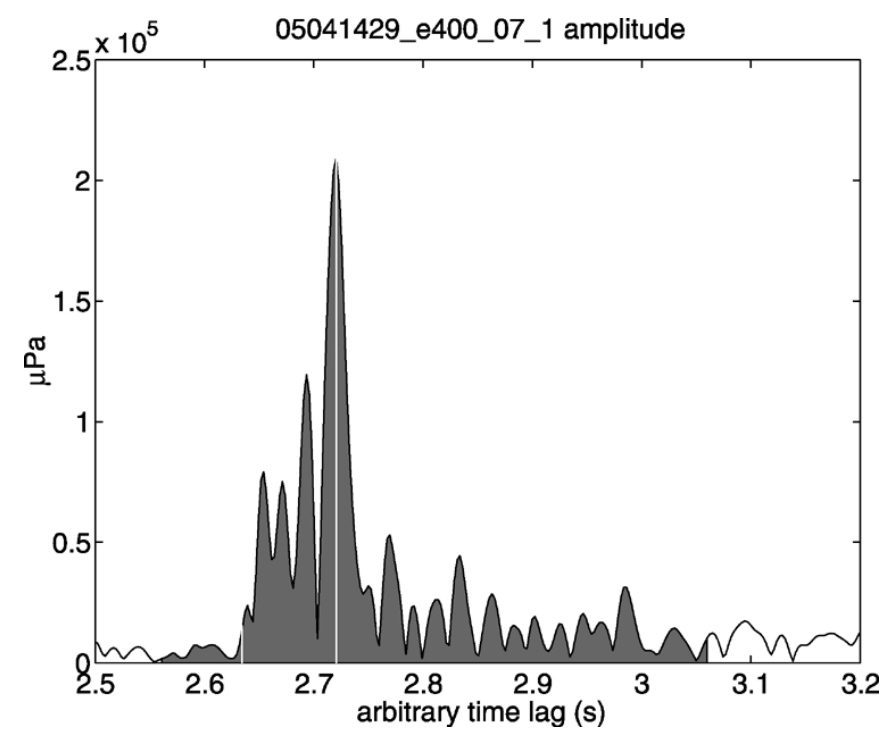

Fig. 6. Matched-filter output pulse of index 20 from the lower panel of the previous figure is shown. Matched-filter gain has been removed. One-half second of the pulse has been highlighted. The white lines in the shaded region indicate leading edge arrival time and peak time.
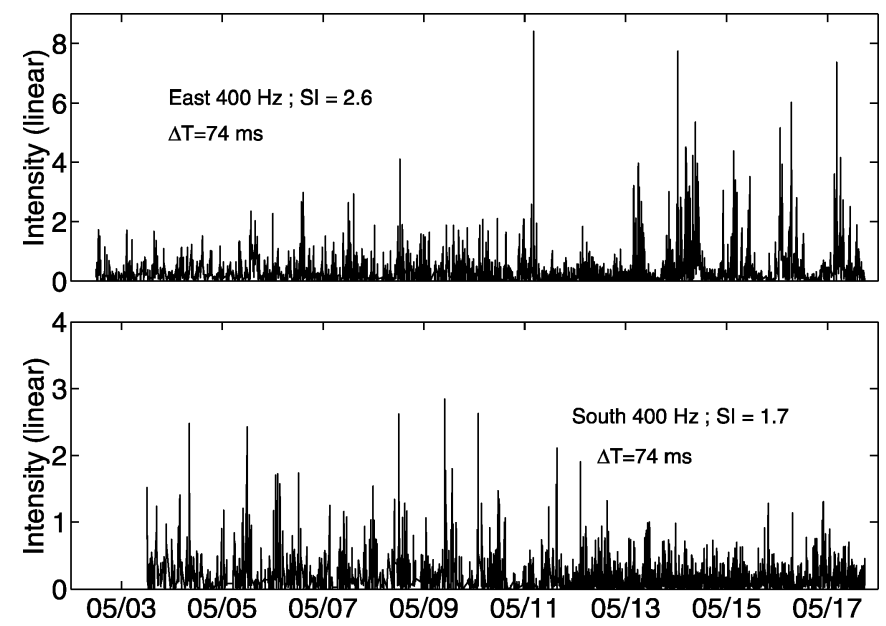

Fig. 7. Intensity time series (arbitrary linear units) seen at VLA hydrophone \#7, $62 \mathrm{~m}$ above bottom, $74 \mathrm{~ms}$ after the onset of each arrival. The top panel shows the receptions from the source to the east, the bottom panel those from the source to the south. The two panels have the same intensity scale.

a deeper than planned site during the experiment because of intense fishing activity at the originally chosen site. This geometry provides less-than-perfect mode filtration capabilities (using the vertical mode orthogonality condition), and also allows energy to pass above the array, which somewhat complicates our analysis of the fluctuations observed. VLA phone 1 was at the top at $42.25 \mathrm{~m}$ depth, with nine phones below at $3.75 \mathrm{~m}$ spacing, and six phones below that at $7.5 \mathrm{~m}$ spacing.

Fig. 5 shows some typical received $400 \mathrm{~Hz}$ pulses. These pulses have $100 \mathrm{~Hz}$ bandwidth and are transmitted as 511 digit, $5.11 \mathrm{~s}$ phase-encoded pseudorandom (M) sequences to reduce source power requirements. The correlated (matched filtered) receptions would look like a 10-ms Gaussian-like pulse in the absence of dispersion and scattering effects by the ocean. However, it is clearly evident that channel dispersion and scattering have lengthened the pulse receptions to the order of 

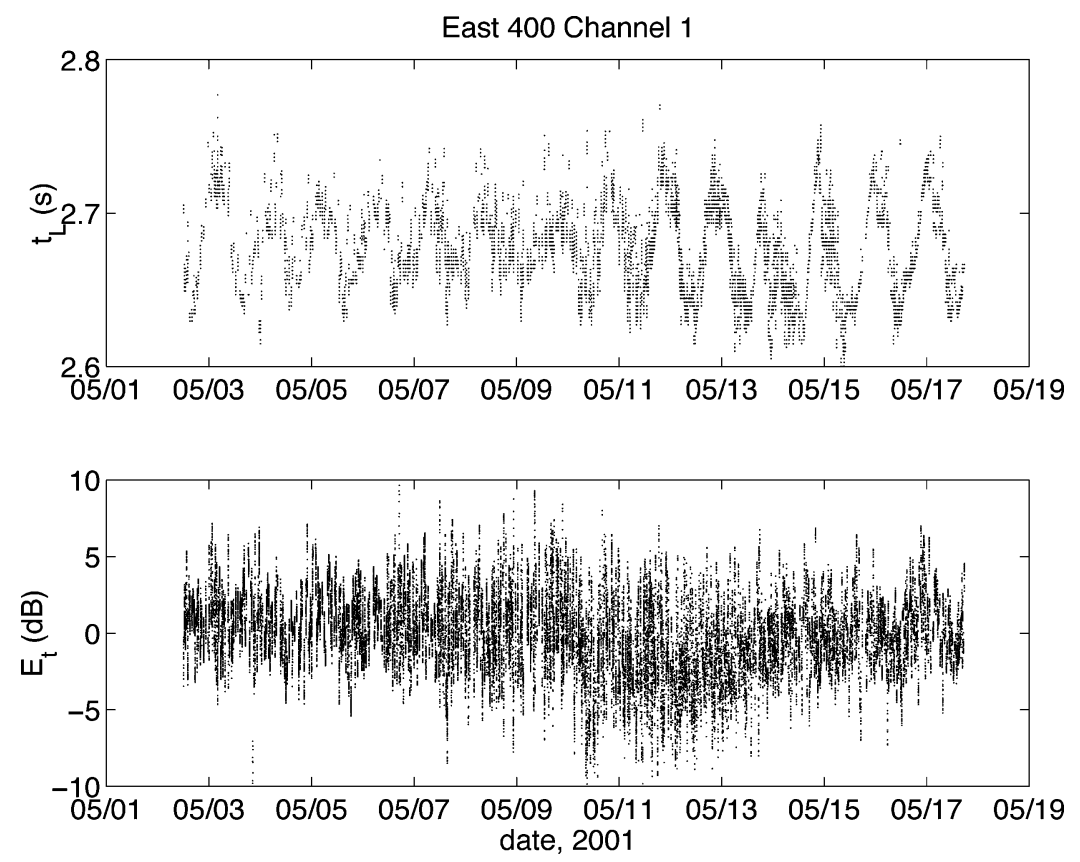

Fig. 8. Top panel shows the time series for the leading edge arrival for the eastern $400 \mathrm{~Hz}$ source receptions at hydrophone \#1 of the WHOI/NPS array. This is the top phone on the VLA, at 42-m water depth. Arrival time shown here is relative to an arbitrary, common beginning time, and is not the total travel time of the pulse. The bottom panel shows the time series of the time-integrated intensity of the pulse arrivals at that hydrophone, with zero decibels taken as the series mean. The standard deviation of $E_{t}$ in decibel units is 2.6.

100 to $200 \mathrm{~ms}$. Moreover, these receptions vary both in space (the vertical coordinate we observe via the VLA, not shown in the figure) and in time (both over clock time or "geotime" and within the reception time of individual pulses). We analyze these structures.

\section{B. Analyses Performed on the Data: Background and Definitions}

We next give a brief description of the physics underlying our data analyses. Intensity fluctuations arise from fluctuation propagation and scattering conditions in the medium. These conditions also influence other aspects of pulses, which we will consider in addition to intensity. Two standard quantities examined when one looks at broadband pulse propagation and distortion are the pulse wander and spread. The wander is simply the change in travel time of the pulse, without any change in the shape of the pulse. This corresponds to adiabatic mode propagation. The spread is the measured duration of the pulse in time, and is associated with coupled mode propagation. These two effects can be closely related despite their distinct propagation physics.

We will examine pulse wander in a cursory fashion using the leading edge of each pulse arrival, as this is a robustly measurable quantity. Although not shown in this paper, pulse spread was also examined using the centroid of each pulse, that is, the "center-of-mass" of the acoustic energy, the time integral of intensity times time, or the first moment of intensity. The spread of the pulse is often twice the distance between the leading edge and the centroid. Different definitions of the spread are also possible, such as the second moment of intensity or the lag time of the intensity peak after the leading edge.

The spread and wander are travel-time related quantities (phase related in the narrowband limit). Our main concern is with amplitude and intensity related quantities. The are many possibilities to consider. Simple quantities are the so-called "point fluctuation" statistics, i.e., fluctuations in amplitude or intensity at a single point hydrophone receiver for a given time after the onset of an arrival. Fig. 6 shows a single pulse arrival and some pulse parameters. The leading edge time (onset) of the pulse will be examined, as will the time and intensity of the peak arrival, each computed for every pulse (i.e., each $5.11 \mathrm{~s}$ during the intermittently spaced transmission periods). The intensity we use for point statistics is that at a fixed time delay after the pulse onset.

Intensity fluctuations statistics will be expressed in terms of the scintillation index, which is the normalized variance of the intensity, given by

$$
S I=\left(\left\langle I^{2}\right\rangle-\langle I\rangle^{2}\right)\langle I\rangle^{-2}
$$

where the means (delineated by brackets) will be taken over periods ranging from $3 \mathrm{~h}$ to $2 \mathrm{wk}$ in this paper.

Another amplitude/intensity statistic we can analyze is integrated intensity, where the integral can be over space (the vertical array), time (the extent of the pulse) or both. This parameter is intended to be directly comparable to the incoherently depth-averaged intensities reported in modeling studies [10], [11]. The time integral of the intensity within the pulse (the energy) at a single hydrophone is a quantity of great interest since square law (energy) detectors are common devices in sonar systems. The double integral over time and the vertical coordinate is also physically interesting, since (if the array spanned the entire water column and a bit of the bottom) it represents the total energy flux passing one point in range. The integral over the vertical array alone is not considered here. Mathematically, the 

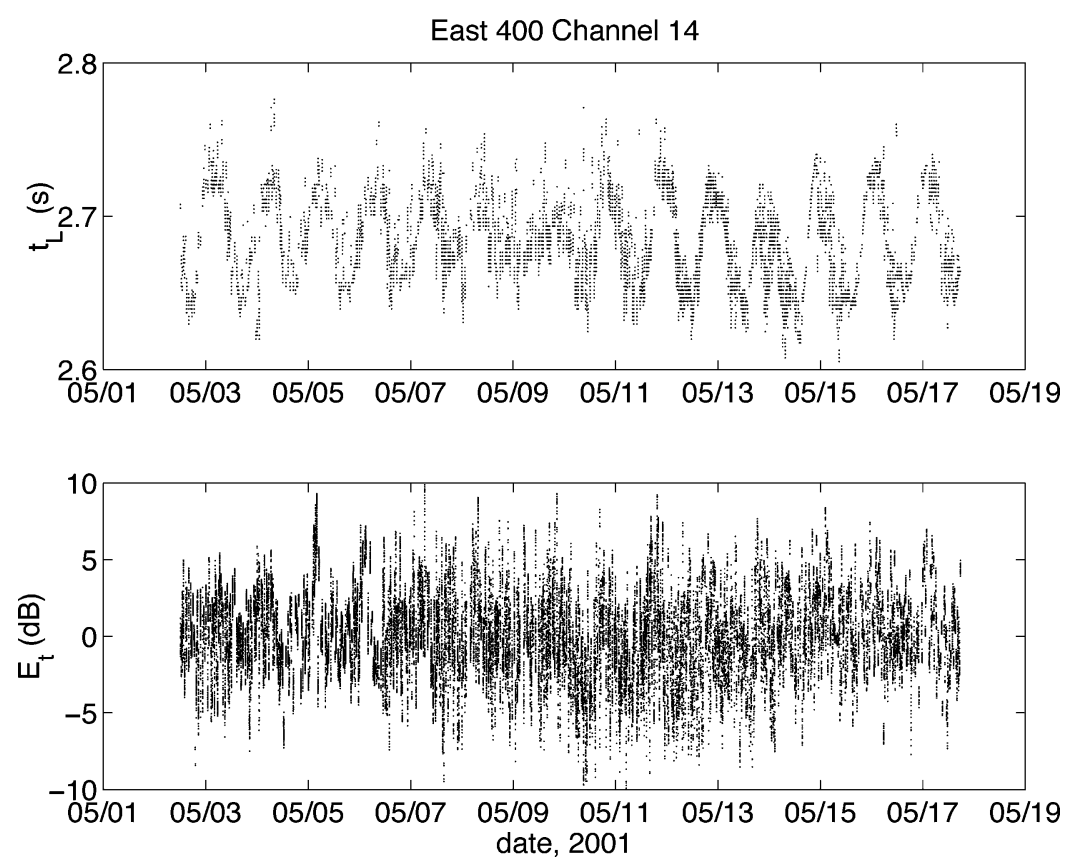

Fig. 9. Similar to Fig. 8, except that data are from hydrophone \#14 of the WHOI/NPS array, which is a near-bottom phone on the VLA, at 113-m water depth (and $11 \mathrm{~m}$ above bottom). The standard deviation of $E_{t}$ in decibels is 2.8 .

time integrated and space-time integrated quantities we will consider are defined by

$$
\begin{aligned}
& E_{t}=\Delta t^{-1} \int_{t 1}^{t 1+\Delta t} a^{2}(t) d t \\
& E_{t z}=N^{-1} \Delta t^{-1} \sum_{n=1}^{N} \int_{t 1}^{t 1+\Delta t} a_{n}^{2}(t) d t
\end{aligned}
$$

where $N$ is the number of hydrophones (16), $a$ is amplitude and $\Delta t=0.5 \mathrm{~s}$. Nearly 40000 pulses from each source were recorded and confidence intervals of all statistics are small.

\section{Intensity Fluctuation Observations}

1) Point Fluctuation Statistics: Fluctuating ASIAEX pulse behavior is now examined using the measures described above. Pulses are analyzed after matched filtering to reconstruct the encoded pulses. Pulse leading edges exceeding an amplitude threshold are found in the data, and, for each pulse, many quantities are computed, including $E_{t}$, peak intensity, time of leading edge, time of peak, centroid time, mean intensity in a window preceding the pulse (noise level), and the signal to noise ratio (SNR, ratio of $E_{t}$ to noise level). Pulses with low SNR or at incorrect times are discarded (some timing ambiguities arose in the recording system).

We will begin with a simple statistic, the point fluctuation scintillation index. Fig. 7 shows intensity time series from the eastern and southern path transmissions, with intensity values taken $74 \mathrm{~ms}$ after pulse onset. From these, SI can be computed after appropriate averaging. One might think that the averaging process would minimize the effects of variations in the pulse shape, which would contribute an unwanted component to the
$S I$ estimate, especially in the region of the onset or tail. However, it was observed in the data that the pulse shape, as quantified by the spread, varied quite significantly even over a few minutes, with the spread changing up to a factor of two, so further care was taken to ensure that a point $(74 \mathrm{~ms})$ was chosen that was always within the pulse.

For the data of Fig. 7, the average intensity is lower for the southern source because the path length is longer. More interestingly, the $S I$ computed for the entire record is higher for the eastern path, 2.6 versus 1.7. These intensity fluctuations can also be expressed as 6.4 and $6.1 \mathrm{~dB} \mathrm{rms}$, respectively. We ascribe this to the smaller average water depth along the eastern path, which effectively forces the acoustic energy to travel closer vertically to the fluctuating thermocline, and thus to interact more strongly with the internal waves, which produce their largest sound speed disturbance within the 10- to 100-m depth range. The deeper southern path, coupled with the ubiquitous downward refracting profile, directs the sound largely away from the perturbed soundspeed region. Even though the southern path is longer, the total amount of scattering appears to be significantly less. We have preliminarily verified this conjecture using CW parabolic equation calculations along the two paths, utilizing measured thermistor records to simulate the internal wave field [T. W. Wang, private communication]. We should note that this result is also consistent with the observation that the horizontal array coherence of the $224-\mathrm{Hz}$ field (transmitted from the southern site) is significantly higher than that of the 300or $500-\mathrm{Hz}$ transmissions from the east [B. Pasewark, personal communication]. While there may be some frequency-dependent coherence length effects, it is our assertion that the increase in the coherence length of the $224-\mathrm{Hz}$ transmission is mainly due to the difference between the southern and eastern paths, and not frequency. 

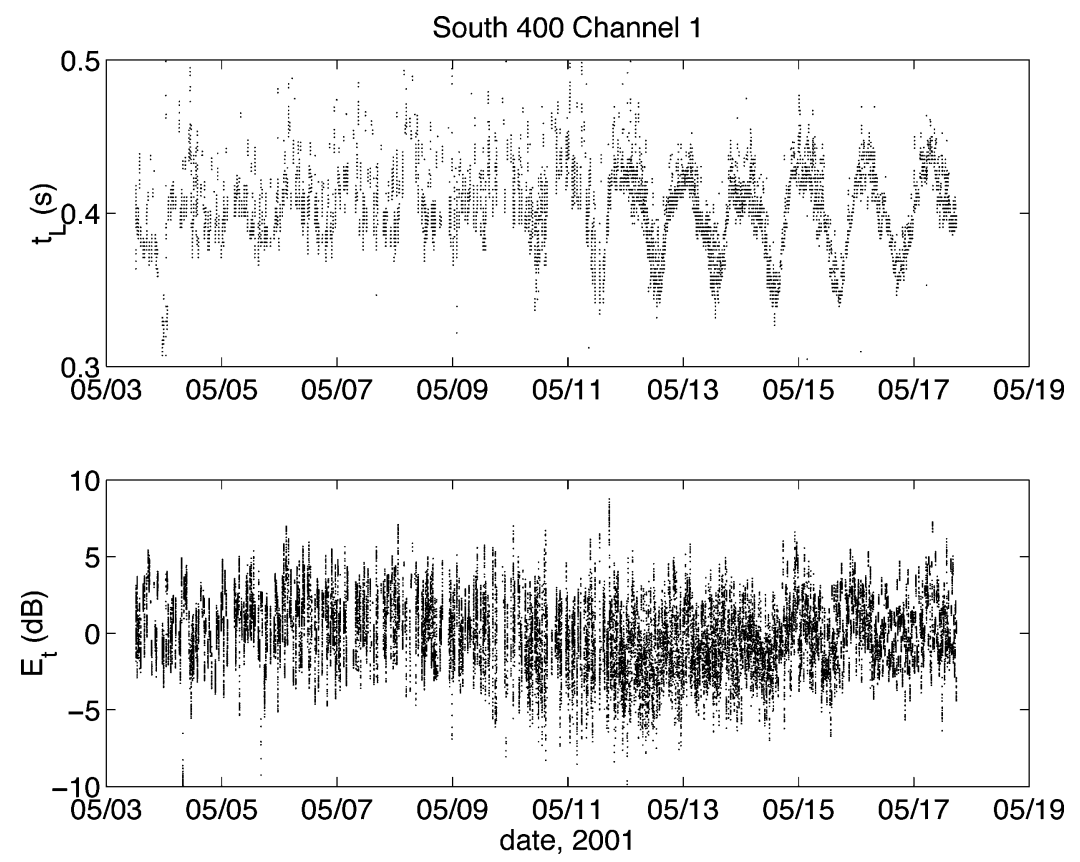

Fig. 10. Similar to Fig. 8, except that parameters of transmissions from the southern source are shown. The standard deviation of $E_{t}$ is $2.4 \mathrm{~dB}$.

2) Time Integrated Intensity Fluctuation Statistics: We next look at $E_{t}$, the intensity integral over the arrival times of the pulses at any given hydrophone. This corresponds roughly to what standard "square law" (integrated intensity) detectors utilize in sonar systems; thus, it is reasonable to consider. It can also be compared to signal levels in monochromatic simulations [10], [11] under the assumption of weak frequency dependence of fluctuation effects.

In Figs. 8 and 9 we show the leading edge arrival time and $E_{t}$ time series for the eastern source receptions at 42 and $113 \mathrm{~m}$ depths. These two are shown together to allow examination of the wander effects with the acoustic intensity fluctuations. In the travel time fluctuations, (top panels of the figures), the diurnal and, in midrecord, the mixed diurnal-semidiurnal internal tide signals are evident. These produce arrival time oscillations of order $100 \mathrm{~ms}$ peak-to-peak amplitude. These are a very large in comparison to the order $10 \mathrm{~ms}$ amplitude internal tidal timing signals seen in the SWARM and PRIMER shallow-water acoustics experiments, which had very similar geometries and configurations [1], [3]. One might note a cleaner looking signal in the second half of the record due to a change in the transmission rates from 88 pulses every half hour to 23 every $10 \mathrm{~min}$. Some pulse groups of each type are missing entirely due to excess noise or to recording gaps.

Turning to the intensity fluctuation statistics, our prime concern here, we see both interesting similarities and differences between the lower panels of Figs. 8 and 9. Both show a considerably noisier appearance than the arrival-time time series, due to the fact that these fluctuations are caused by both the internal tides and the high-frequency internal waves, with the latter probably being the dominant effect, as shown later. The latter effect is also discussed in detail in another paper [12]. A second effect to note is that the receptions at hydrophone \#1 (Fig. 8, lower panel) have a distinct reduction in mean level from 10-13 May. Adopting a ray viewpoint, this reduction can be explained by surface interaction of some ray paths in the pulse during the high-wave period of typhoon Cimaron. The surface interaction scatters a considerable portion of the energy to higher angles, where it is subsequently lost to the bottom. This dip in the intensity is not as notable for the deep arrivals (lower panel of Fig. 9) because of the fact that deep phones receive a smaller fraction of high angle (high mode) energy with respect to the total received energy than do higher phones. This is due to the near bottom source and receiver placements in the downward refracting profile that favors low angle (low mode) arrivals. The low angles do not have appreciable surface interaction, so the deep-phone receptions are less attenuated by the typhoon waves. The sound-speed profile in the area was modified by the typhoon passage, but the change persisted until the end of the experiment, whereas the signal level recovered on May 13, so the hydrographic change does not explain the temporary drop in signal level.

The variance of $E_{t}$ is comparable for the higher and lower receivers (2.6 versus $2.8 \mathrm{~dB}$ ). Even though the signals differ in their amount of surface interaction, it appears that the amount of interaction with the high-frequency internal waves, appearing as a continual high-frequency intensity fluctuation, is roughly equal for both receivers, which is perhaps not surprising because the internal wave field perturbation of the soundspeed spans most of the water column for the eastern path. (But not the southern path, as shown later.)

We next look at the southern transmissions to the same two VLA hydrophones, and contrast them with their eastern counterparts. These time series are shown in Figs. 10 and 11 and are in the same format as Figs. 8 and 9. Though there are notable similarities between figure pairs $8 / 10$, and $9 / 11$, there are also some notable differences, which we describe here. To begin with, the standard deviation of $E_{t}$ is least for the southern transmissions to hydrophone \#1 (the upper phone, Fig. 10) than for the other configurations, indicating that this configuration might have had 

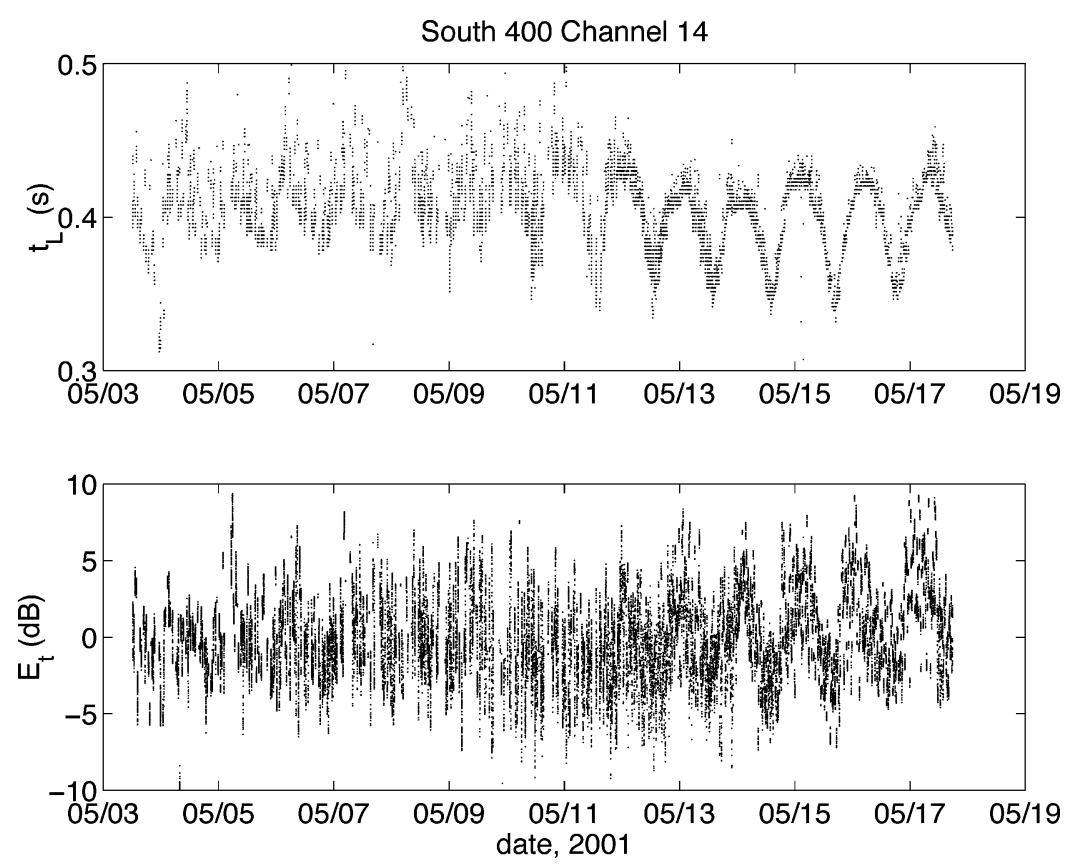

Fig. 11. Similar to Fig. 9, except that parameters of southern source signals are shown. The standard deviation of $E_{t}$ in dB units is 3.0.

the least interaction with the internal wave field. This agrees with parabolic wave equation calculations, which show that energy from the southern source has traveled close to the bottom, away from the internal wave field, for most of the upslope path. However, the deeper hydrophone (Fig. 11) shows the greatest of the four illustrated fluctuations. The internal wave perturbations to the soundspeed extend typically to $\sim 100 \mathrm{~m}$ water depth, with paths reaching above this depth sensing the waves, and deeper paths not. Clearly the sound arriving at the deep phone has interacted with the thermocline and is not isolated from it for the entire path [12]. We also note that in the latter third of the record, particularly for the deeper receiver (Fig. 11), an internal tide signal appears in the amplitude fluctuation. This may be due to the internal tides moving the energy up and down at the receiver location or altering conditions at the source, rather than due to high-frequency wave scattering, as the high-frequency internal waves are weak at the beginning and end of the record, but strong in the middle. We finally note that the hydrophone \#1 receptions of southern signals show some loss, again attributed to the typhoon, but hydrophone \#14 shows little effect. This effect is weaker for the southern path than for the eastern path, possibly because the southern path energy generally stays deeper than the eastern path and thus interacts less with the surface.

Power spectra of the $\log$-energy $\left(\log E_{t}\right)$ and the leading edge time $t_{L}$ for hydrophone 8 signals from the eastern source are shown in Fig. 12. The Lomb-Scargle periodogram method is used to compute the spectrum, and Lomb normalized periodograms are shown [13]. Two frequency bands are shown for each parameter. Both the energy and the leading-edge arrival time show energy near the O1 principal lunar tidal period $(25.82 \mathrm{~h})$, with the peak being more pronounced for $t_{L}$. The spectrum of $\log E_{t}$ shows more activity at the highest frequencies measured than does the spectrum of $t_{L}$ [compare Fig. 12(b) and (d)], the effects of which can be seen in Fig. 13.
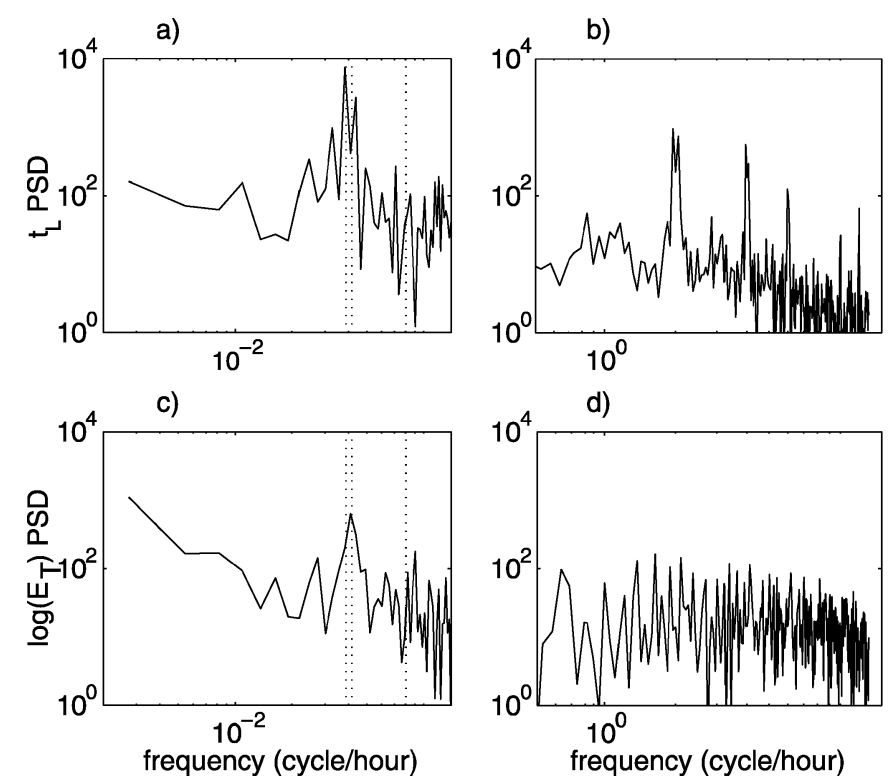

Fig. 12. Power spectral densities (Lomb normalized periodograms) of leading edge time $\left(t_{L}\right)$ and energy $\left(\log E_{t}\right)$ are plotted. (a) and (b) show spectra for $t_{L}$ within low-frequency and high-frequency bands, respectively, with a slight gap, while (c) and (d) show spectra for $E_{t}$ in the same bands. The O1, K1, and M2 tidal frequencies are indicated with dotted lines (25.82-, 23.93-, and 12.42-h periods).

The spectrum of $t_{L}$ also shows activity peaks at periods related to the burst sampling intervals of 30 and $10 \mathrm{~min}$ [Fig. 12(d)].

3) Time and Depth Integrated Statistics: We can also examine the fluctuation statistics of time and space integrated intensity $E_{t z} \cdot E_{t z}$ is an experimental proxy for the energy flux past a range point, with the caveat that our array did not span the entire water column, so that the vertical spatial integral is incomplete. Fig. 14 shows the time series of integrated intensity $E_{t z}(t)$ from both sources. As seen in the point statistics, the southern 

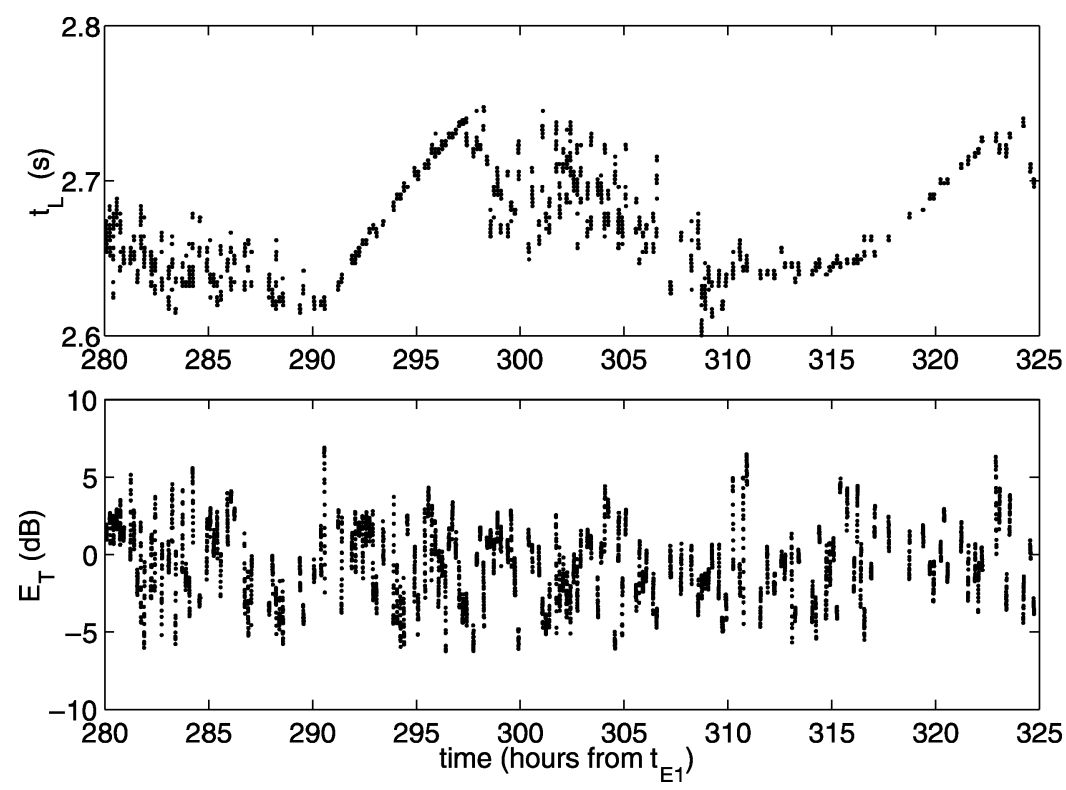

Fig. 13. Detail of eastern source pulse arrival parameters $\left(E_{t}\right.$ and $\left.t_{L}\right)$ whose spectra are shown in the previous figure. The data shown begin at $0401 \mathrm{UTC}$ on 14 May.
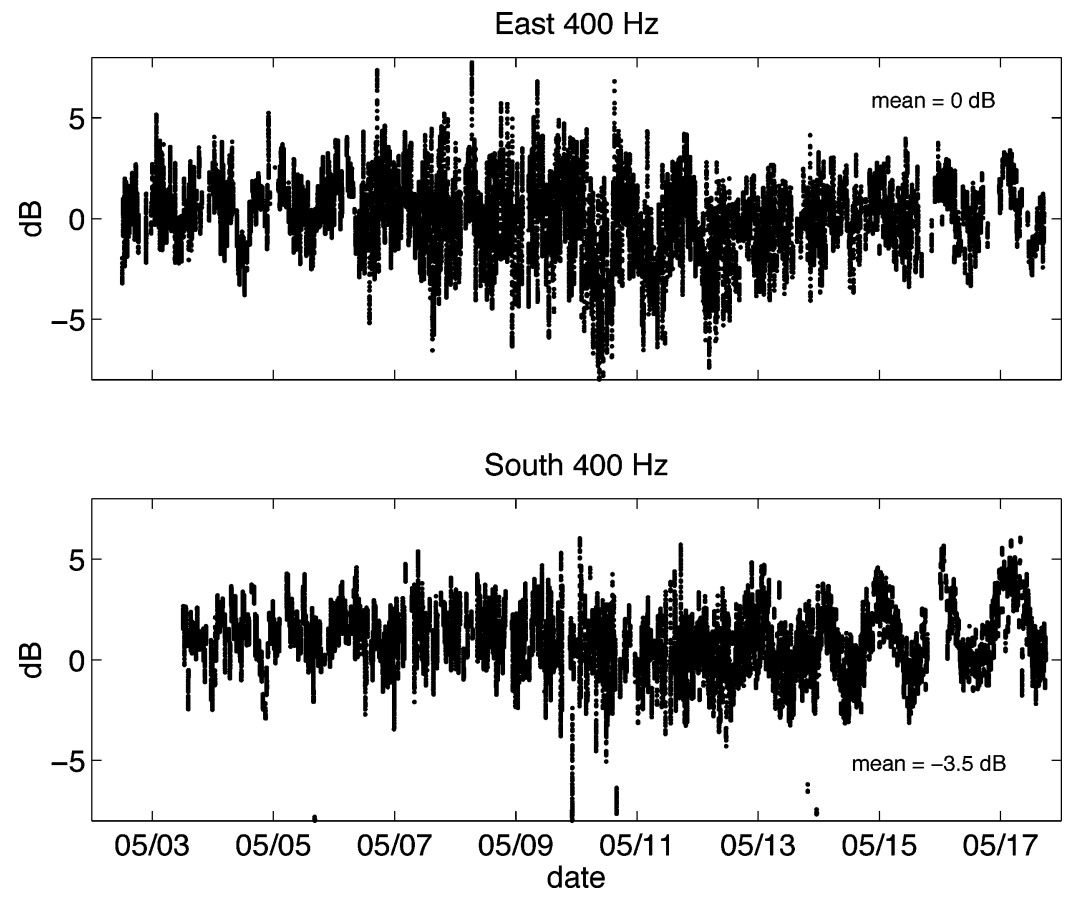

Fig. 14. Space-time integrated intensity $E_{t z}$ for the eastern path (top panel) and southern path (bottom panel) are plotted. The standard deviations are 2.00 and $1.65 \mathrm{~dB}$, respectively. The internal tide dependence of the intensity is clearly seen. The southern path mean intensity is $3.5 \mathrm{~dB}$ lower than that of the eastern path.

path has a lower average intensity, due to the difference in path length. We again see the interesting intensity dip in the upper panel due to surface waves from hurricane Cimaron passing through the site from roughly 10 through 13 May. The explanation for this is the same as for the dip in time-integrated intensity $E_{t}$. For the eastern path (top panel), the sound energy interacts more significantly with the upper part of the water column, including the surface, inducing more scattering and scattering losses. For the southern path (lower panel), the sound has significantly less interaction with the upper water column, and thus has more steady mean intensity.
There is a strong internal tidal signal in both panels of Fig. 14, plus high-frequency effects consistent with modeling of sound passing through moving internal-wave packets. If the vertical integral was over the complete water column (and some of the bottom), the fluctuations of Fig. 14 would represent time-varying losses to the bottom (in a 2-D approximation that ignores out-of-plane scattering). However, since the VLA only extends from the bottom $(124 \mathrm{~m})$ to $41 \mathrm{~m}$ depth, there is a possibility that the detected energy fluctuation is due to energy occasionally moving above the array. Precisely this situation was examined in the PRIMER experiment [14] and 
(a)

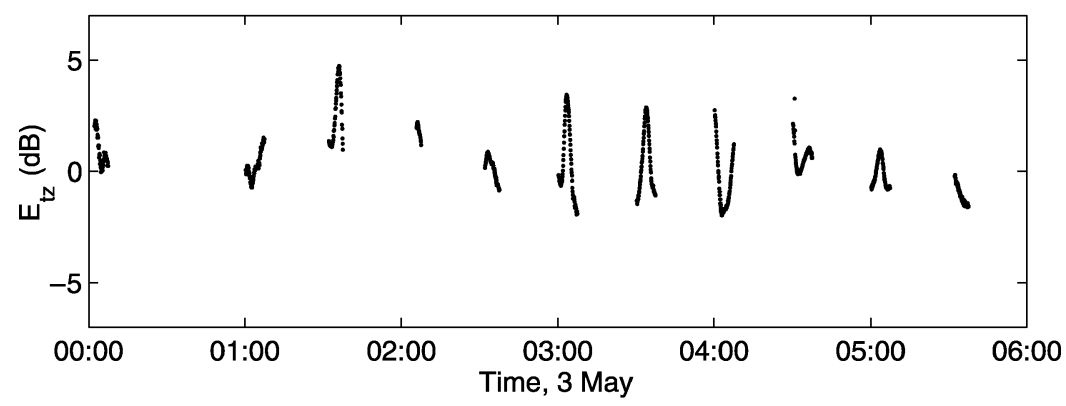

(b)

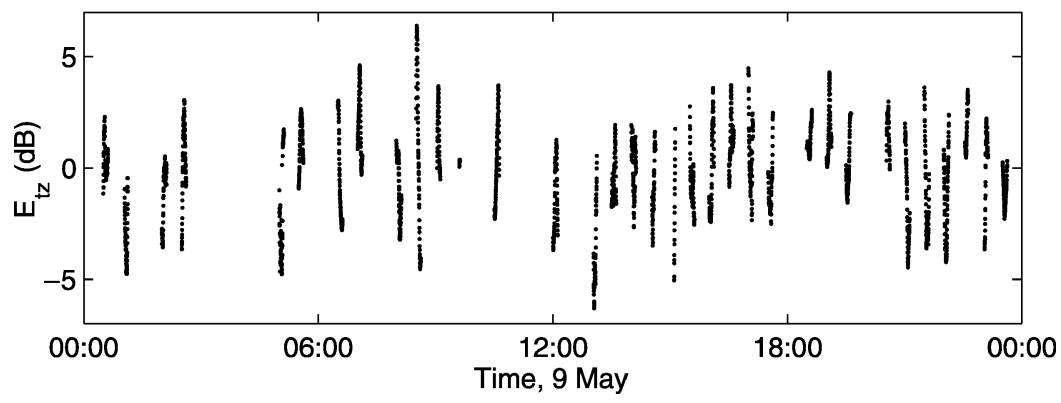

Fig. 15. Details of $E_{t z}$ for the eastern source are shown. (a) Data from $6 \mathrm{~h}$ of 3 May are shown. (b) Data from $24 \mathrm{~h}$ of 9 May are shown. The transmission periods are roughly $7.5 \mathrm{~min}$ long.
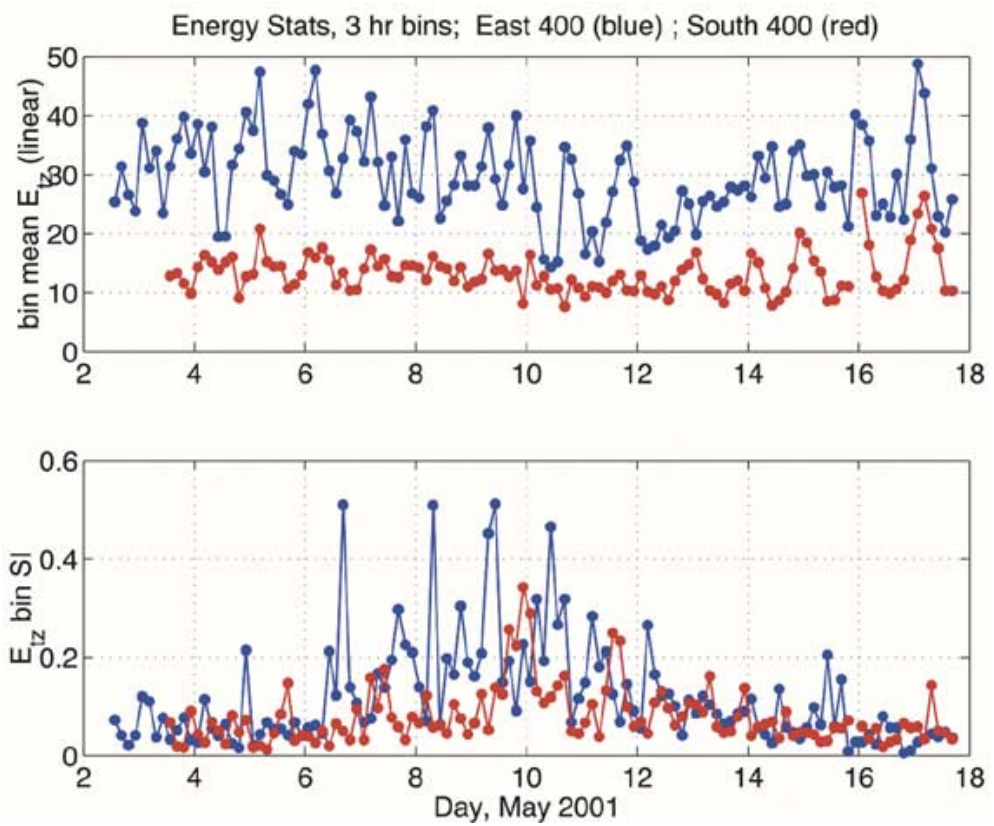

Fig. 16. Depth and time integrated intensity statistics. The upper panel shows the mean $E_{t z}$ in 3-h long bins, from both the eastern source (blue) and the southern source (red). An order of 3.5-dB difference in levels is noted, due to the path length difference. The lower panel shows the variance of $E_{t z}$, in the bins, normalized by the 3 -h mean values to form scintillation indexes, again for both sources.

it was seen in that case that the primary cause of the detected fluctuations was energy moving vertically over time, and not time-varying bottom loss. However, calculations published elsewhere support the notion that time-varying bottom loss due to high-frequency internal waves is indeed occurring during ASIAEX [12], and the same may be true for internal tides. The difference is likely due to the higher strength of the ASIAEX internal wave field.

For the purpose of highlighting the temporal behavior, Fig. 15 shows $E_{t z}$ for eastern signals for two short time in- tervals. The vertical stripes are very similar to depth-averaged energy variability seen in simulated monochromatic sound propagation through moving packets of steep nonlinear internal waves [10], [11], [15]. This suggests, but does not prove, that the mechanisms by which moving wave-packet sound-speed perturbations induce intensity fluctuations, namely, temporally variable mode coupling, are responsible for the observed ASIAEX $E_{t z}$ fluctuations.

To look at the steadiness of the fluctuations, we next look at low-frequency modulations of the space-time integrated inten- 


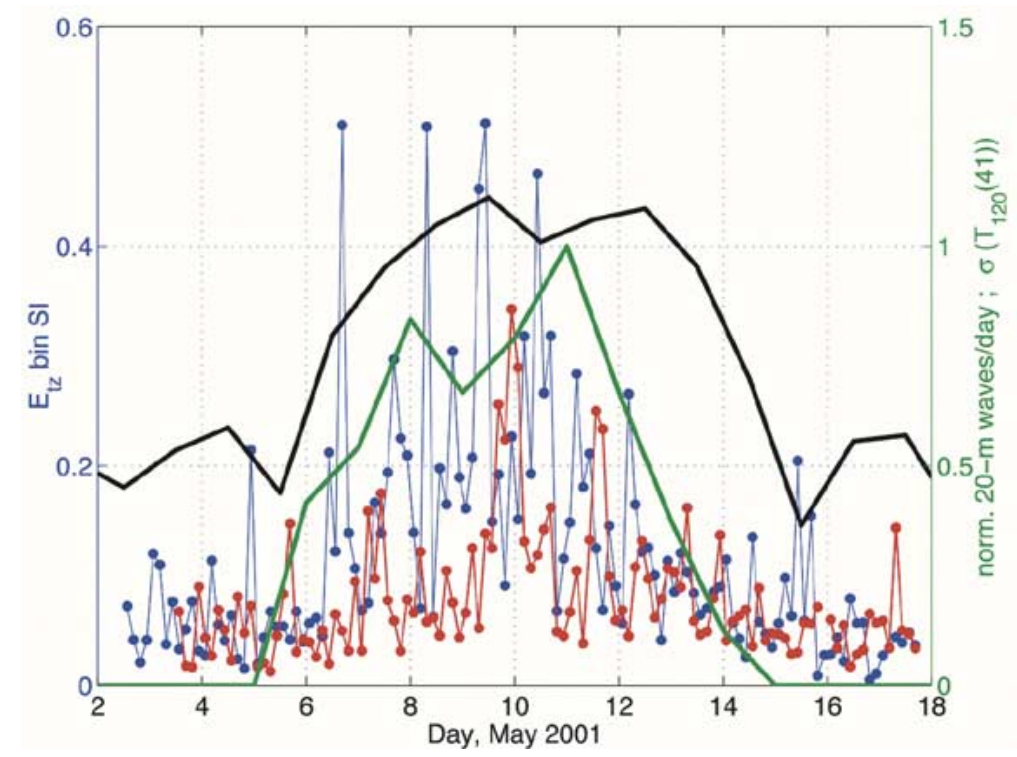

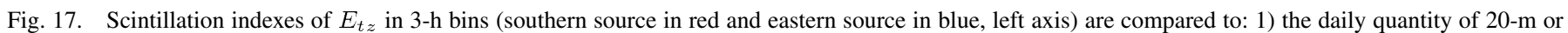

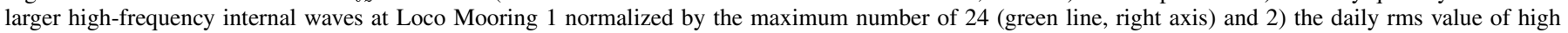
frequency $T$ fluctuations (periods less than $8 \mathrm{~h}$ ) measured at approximately $41-\mathrm{m}$ depth at the 120-m site (black line, right axis).

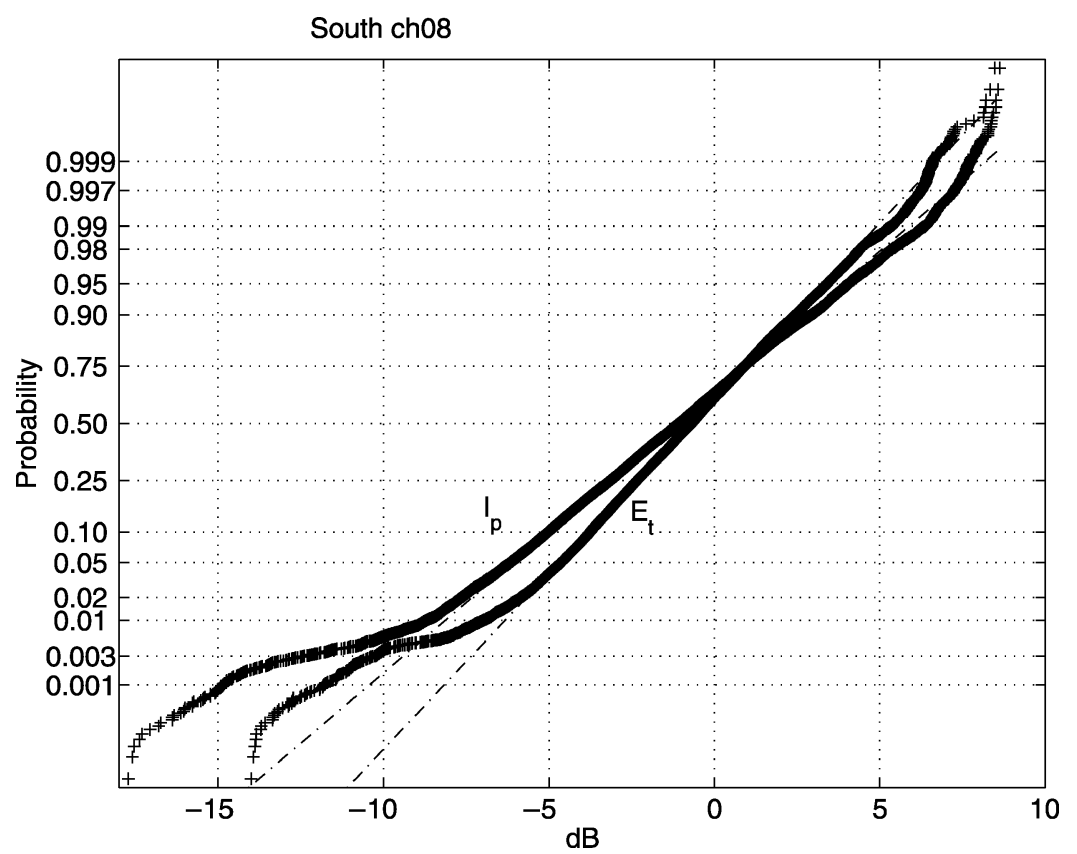

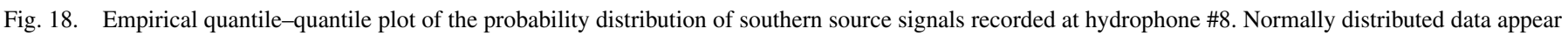

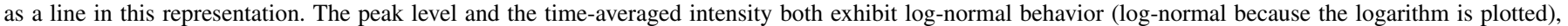
except for the lowest one percent of data which have anomalously low intensity with respect to log-normal.

sity fluctuations, using 3-h binned results. Fig. 16 shows 3-h averaged $E_{t z}$ in the upper panel and variance of $E_{t z}$ in the lower panel, with variance plotted as scintillation index using normalization values taken from the upper panel. The upper panel shows subtidal and tidal variability, and the lower panel shows low-frequency modulations of high-frequency effects. There is a roughly factor of two intensity difference $(\sim 3.5 \mathrm{~dB})$ between the levels received from the two sources, evident in the upper panel. In the lower panel, we see a higher variance in the eastern source path, as was noted for single-depth results. We also see what appear to be once per day peaks in the variance from 7 to 12 May, a time when high-frequency internal wave activity was prominent. Although the once-per-day peaks do not correspond with passage of packets over the eastern source or the receiver, and are thus not yet fully explained, we have observed correlations of the intensity variances with the measured number density of large amplitude internal solitary-type waves, and with the measured statistics of high-frequency temperature (sound speed) perturbations. We show this in Fig. 17 by plotting the daily number of $20 \mathrm{~m}$ amplitude internal waves of period 10 min or less and plotting the daily standard deviation of middepth temperature $T$, along with the scintillation indexes. Some correlation is apparent, as is a superimposed random variability of the intensity variance, which is consistent with previous results 
(a) E-ch08
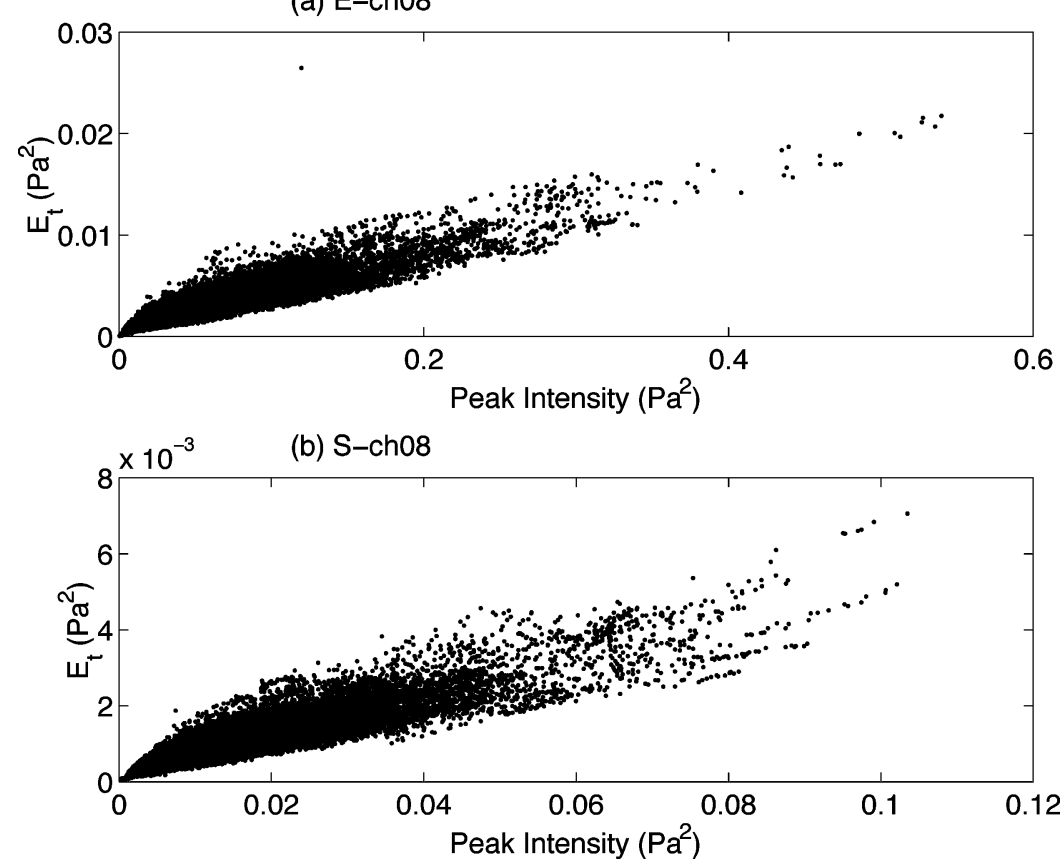

Fig. 19. Time-integrated intensity is plotted versus peak intensity for signals recorded at hydrophone \#8 from eastern (a) and southern (b) sources.

[10], [11], [15] that show complex relationships between wave packet location in the source-receiver transect and the resulting packet-induced intensity variability.

The highest levels of 3-h $E_{t z} S I$ (above 0.2 ) occur when large waves are most plentiful. (Here, we have made the assumption that the temporal behavior of the number of waves present at Loco Mooring 1 is comparable to that along the propagation path. This is allowable because the temporal behavior of the wave census does not vary strongly as a function of position over the measured domain, although there is some trend in wave activity versus depth at fixed time.) Furthermore, the apparent background high-frequency (supertidal) temperature perturbation level of $\sigma(T)=0.5^{\circ} \mathrm{C}$, which is typical of periods with no large internal waves, seems to be associated with 3-h SI values of less than 0.1. High $\sigma(T)$ values correspond imprecisely with high $S I$ values.

4) Peak Level Fluctuations: The level of the highest peak intensity attained within each pulse is subject to a number of controlling factors, including relative array movement through a spatial mode interference pattern, true energy loss, and changes due to pulse spread at fixed energy. Discrimination between these factors is beyond the scope of this paper, but we will present selected peak level statistics. Time series of peak level $I_{p}$ appear similar to $E_{t}, E_{t z}$, and point intensity time series and will not be shown.

Fig. 18 shows the probability distribution function of $\log \left(I_{p}\right)$ fluctuations around their mean level $(0 \mathrm{~dB}$ in the figure), along with a similar plot for $\log \left(E_{t}\right)$, both taken from southern source signals recorded at hydrophone \#8. The behavior of each is approximately log-normal, with the peak level having a higher standard deviation $3.16 \mathrm{~dB}$ compared to $2.51 \mathrm{~dB}$ for $E_{t}$. Next, consider relative behavior of these same two quantities $I_{p}$ and $E_{t}$. These are plotted against each other in Fig. 19 using data from the same hydrophone. Signals from both sources are

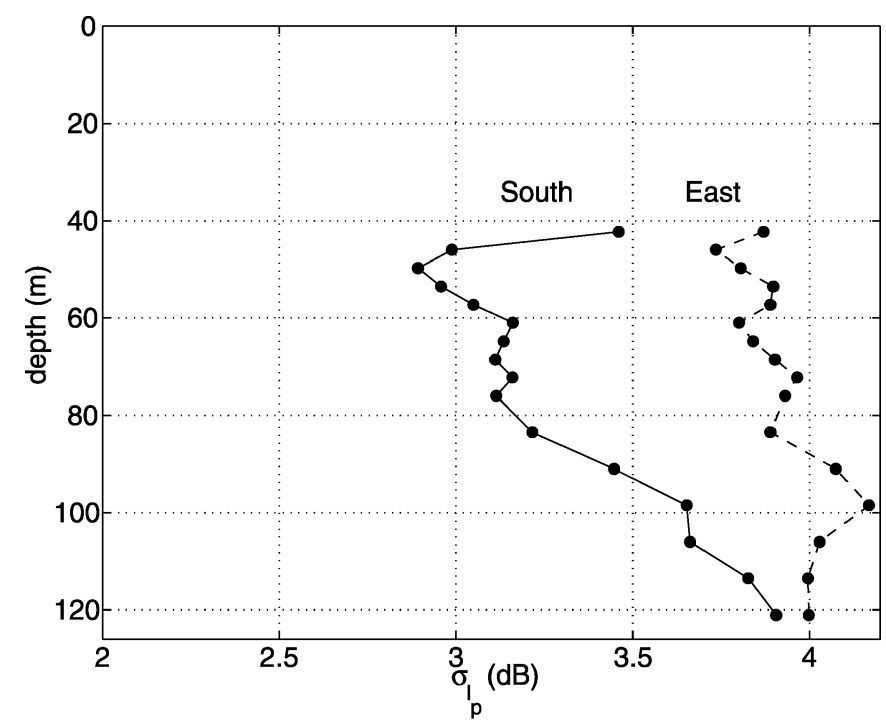

Fig. 20. Standard deviation of the logarithm of peak intensity is plotted versus hydrophone depth for pulses from each of the two sources, southern (left) and eastern (right).

shown. There is a clear tendency for the two quantities to be correlated. However, there is considerable spread in observed $I_{p}$ values for a given $E_{t}$ and vice versa. The south data show a larger spread in this representation, which uses different scaling for the two plots. The relationships between these and other quantities, and their relationship to each other, bears further investigation.

Fig. 20 shows the depth distribution of the standard deviation of $I_{p}$. The variability is greater near the bottom. We have no simple explanation for the depth dependence at this time. These standard deviations are lower than the 5.6-dB value that intensity with an exponential distribution would have (those distributions have $S I$ of one, also) [16]. Lognormal distributions of 

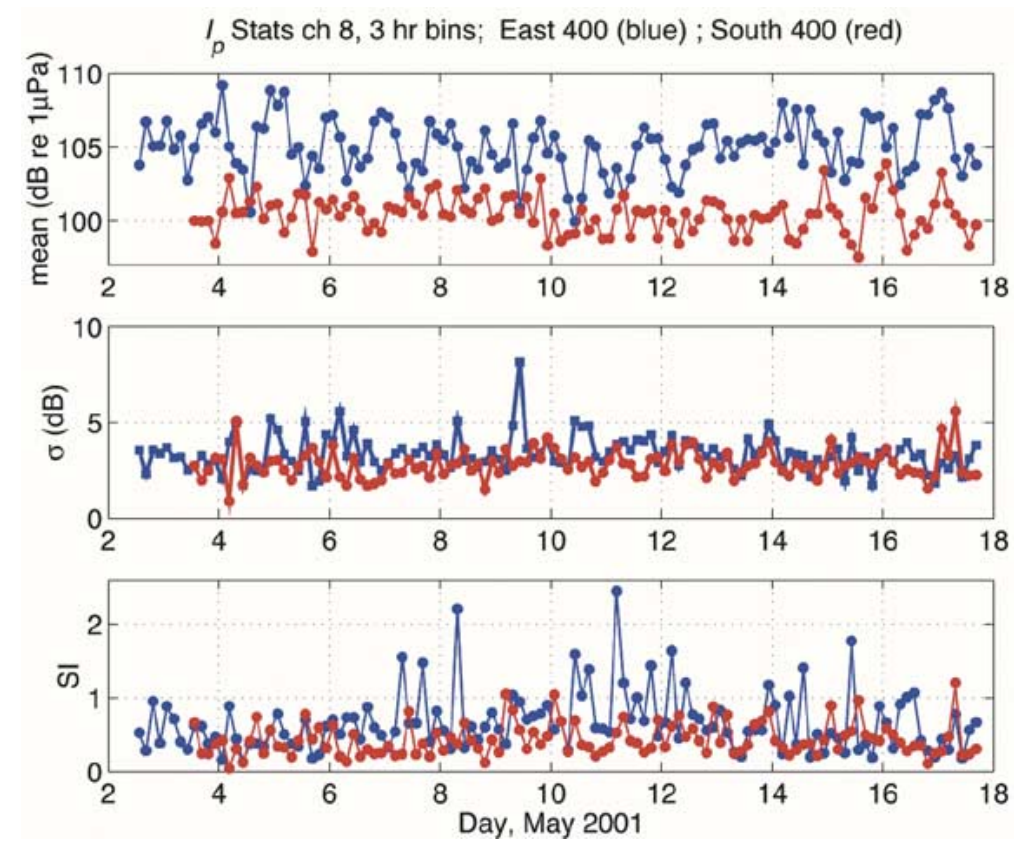

Fig. 21. Statistics of peak intensity level computed independently in 3-h long bins are shown. Date are from hydrophone \#8. Top: mean of the intensity. Center: standard deviation of the logarithm of intensity. Bottom: scintillation index.

intensity can have a variety of rms decibel values and a lognormal distribution with $S I$ equal to one would have a standard deviation of approximately $3.6 \mathrm{~dB}$, comparable with some of our values.

Finally, we consider the temporal variability (nonstationarity) of the peak fluctuation statistics. Fig. 21 shows statistics of $I_{p}$ computed in 3-h bins, the same binning used to investigate $E_{t z}$ fluctuations. A comparison of Figs. 21 and 16 shows that $I_{p}$ has higher scintillation indexes than $E_{t z}$, which makes sense because $I_{p}$ is not an averaged quantity as is $E_{t z}$. The mean and the two variance measures that are shown (standard deviation of the decibel values, and scintillation index) are all quite variable throughout the record for both propagation paths. The eastern path shows a greater change in behavior of $I_{p}$ statistics in response to the spring-neap tidal and nonlinear internal wave cycles (Fig. 4) than does the southern path. This distinction between the paths is also seen for $E_{t z}$ (Fig. 16). The eastern path, in addition to having the largest changes in statistical behavior, also has the highest 3-h scintillation indexes of peak intensity, exceeding two for a few of the 3-h long intervals (Fig. 21, lower panel).

\section{Temporal Correlation Scales}

We next look at the temporal correlation (or equivalently, decorrelation) scales of the fluctuating received energy. We consider the normalized time-lagged autocovariance function (the autocorrelation) of $E_{t z}$, called $R_{E}$, computed independently for each day of the experiment. This investigation of the decorrelation of the signal energy is complementary to the work of Mignerey and Orr, who studied the temporal correlation function of ASIAEX acoustic field vectors $F(z)$ with $F$ normalized to have unit magnitude, so that they were investigating field structure rather than energy [5]. The results for $3 \mathrm{~d}$ of southern path transmissions are shown in Fig. 22. It is seen that the decorrelation time varies substantially from day to day. Moreover,

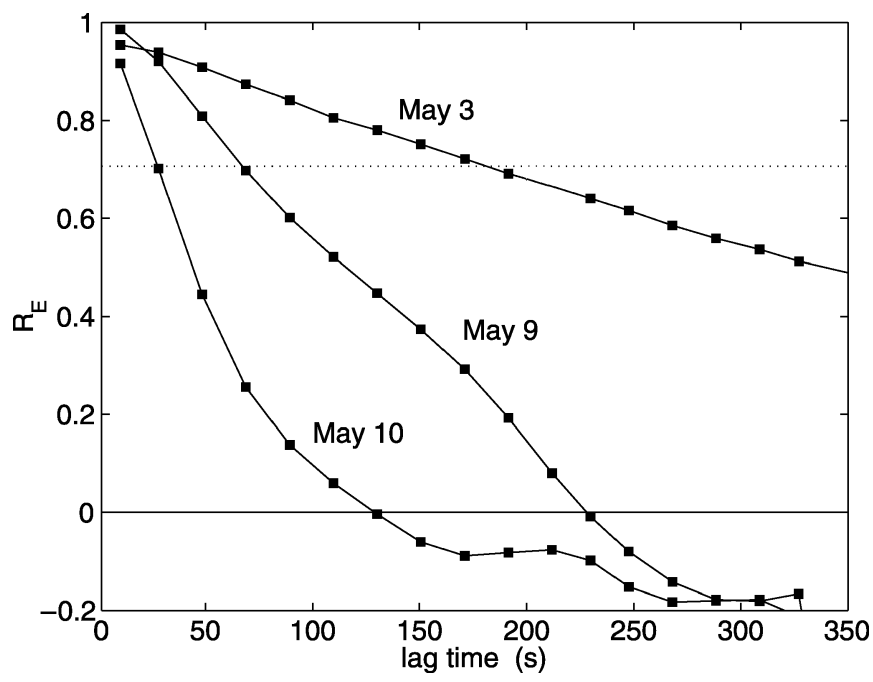

Fig. 22. Temporal correlation functions of $E_{t z}$ for each of $3 \mathrm{~d}$ of the experiment, southern $400-\mathrm{Hz}$ source.

the shortest decorrelation times correspond to the period of the highest solitary internal wave activity. Finally, these time scales are on the order of 1 to $3 \mathrm{~min}$, similar to previous shallow-water results [17].

Fig. 23 shows the time at which the correlation function drops to the level 0.707 , named $T_{R}$, again computed for each day. For both propagation paths this decorrelation time scale is smallest during the middle period of the experiment, when the internal wave activity is highest, and is larger during the initial and final segments of the experiment. There is also an interesting geometric path dependence. For the southern path, over which the internal wave scattering is reduced by ducting of the sound near the bottom, away from the depths of highest sound speed fluctuation, $T_{R}$ is quite high at the initial and final periods of the experiment, approximately $200 \mathrm{~s}$. As we shall see, only when 

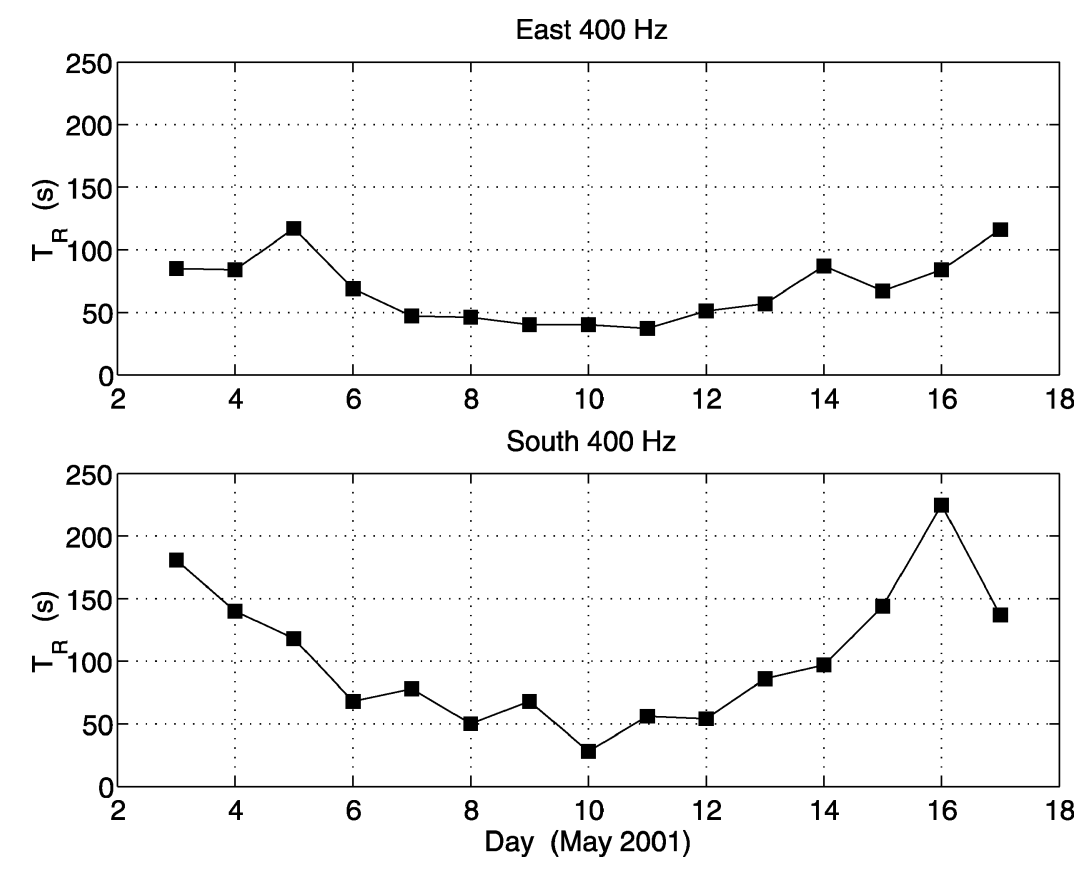

Fig. 23. Daily decorrelation times for signals from the eastern (upper panel) and southern (lower panel) sources.
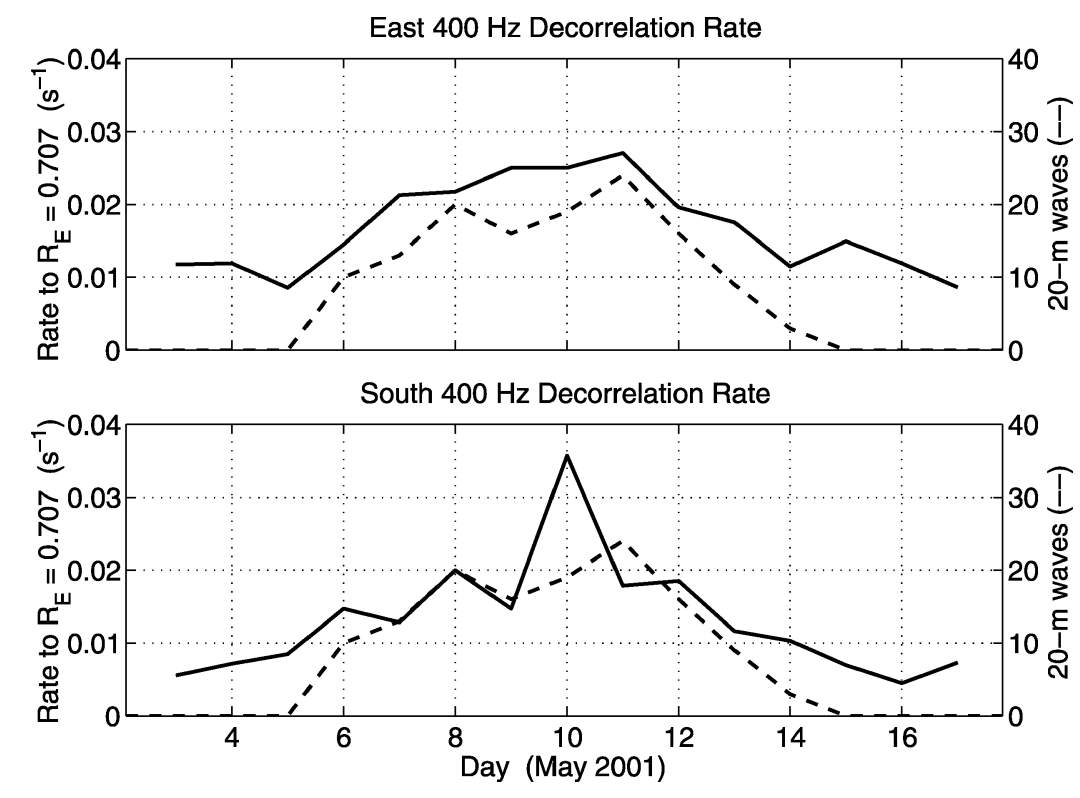

Fig. 24. Decorrelation rate (inverse of decorrelation time) computed daily is plotted (solid line) for the eastern source (top panel) and southern source (lower panel). The daily number of $20 \mathrm{~m}$ amplitude waves from Fig. 17 is also plotted (dashed lines).

the internal wave activity is at its peak does the correlation time drop to $50 \mathrm{~s}$. On the other hand, the eastern source path shows only approximately $120 \mathrm{~s}$ decorrelation time during the low internal wave activity period, dropping to near $50 \mathrm{~s}$ for most of the experiment. This is consistent with penetration of the acoustic energy higher into the water column for this path than for the southern path.

Finally, we look explicitly at the relationship between the internal wave activity and the acoustic decorrelation time. Fig. 24 shows the similarity of the daily quantity of larger internal waves (20 $\mathrm{m}$ amplitude) to the acoustic decorrelation rate (inverse of the decorrelation time shown in Fig. 23). A very good overall agreement is seen. The agreement is not as good if the daily count of smaller internal waves of $10 \mathrm{~m}$ amplitude is considered (not shown), suggesting that the largest internal waves are the principal contributors to reduction of the temporal decorrelation time.

\section{CONCLUSION}

Fluctuations of $400-\mathrm{Hz}$ pulse signals propagated over two paths in the SCS have been presented. One path was $20.6 \mathrm{~km}$ in a roughly along-shelf orientation (the eastern path), and one was $31.3 \mathrm{~km}$ long in an upslope transmission fashion (the southern path). Signals were recorded with a vertical line array of 16 hydrophones. Intensity fluctuations were mainly considered. 
Point statistics show very strong fluctuations, with scintillation indexes well over the saturation value of one, indicating some structure in the scattering process rather than a simple randomness, comparably viewed as an effectively reduced number of interfering multipaths [18]. Correlated signal contributions (rather than uncorrelated) can reduce this effective number, or the number can be smaller in actuality. In either case, a small effective number of interfering signals gives elevated intensity variance, potentially higher than the saturation value of one, because the resulting field exhibits strongly focused peaks separated by extended regions (or time periods) with far less energy. For signals at VLA hydrophone \#7, the eastern path had higher point-computed scintillation index than the southern path, 2.6 versus 1.7 .

Time integrated pulse energies display variability, including loss at the time of typhoon Cimaron that may be due to the roughened sea surface. This attenuation is more evident in shallower eastern path than for the southern path, in agreement with expectations of bottom-trapped propagation dominating the offshore two-thirds of the southern path [12].

Time- and depth-averaged intensity, meant to quantify acoustic energy, but doing so imperfectly because of a lack of hydrophones in the upper $40 \mathrm{~m}$, showed the rapid fluctuations previously seen in modeling studies of sound passing through steep internal waves. The statistics of the fluctuations changed over the spring-neap tidal cycle, with higher scintillation indexes during periods with more plentiful high-amplitude internal waves and stronger temperature perturbations. The correlation time scale of this averaged intensity ranges from a low of $30 \mathrm{~s}$ to highs of over $200 \mathrm{~s}$, and follows the same tidal cycling as the scintillation index.

Statistics of the peak levels attained by the individual pulses were also examined. These had higher variance near the bottom than at shallower depths for both southern and eastern propagation paths. Spring-neap cycling can be seen in eastern path fluctuations but are not as evident in southern path fluctuations.

Overall, the results are consistent with variable coupled-mode propagation along both paths. The southern path possessed a large volume of cold water beneath the thermocline not possible for the shallower eastern path, so that the ducts along the two paths differed in a way that affected some of the fluctuation processes and statistics.

\section{ACKNOWLEDGMENT}

The authors would like to thank the ship's crews, engineers, students, and scientists listed in the introductory paper in this issue. This is WHOI contribution 10929.

\section{REFERENCES}

[1] J. R. Apel, M. Badiey, C.-S. Chiu, S. Finette, R. Headrick, J. Kemp, J. F. Lynch, A. Newhall, M. H. Orr, B. H. Pasewark, D. Tielbuerger, A. Turgut, K. von der Heydt, and S. Wolf, "An overview of the 1995 SWARM shallow water internal wave acoustic scattering experiment," IEEE J. Oceanic Eng., vol. 22, pp. 465-500, 1997.

[2] B. H. Pasewark, S. N. Wolf, M. H. Orr, and J. F. Lynch, "Acoustic intensity variability in a shallow water environment," in Impact of Littoral Environmental Variability on Acoustic Predictions and Sonar Performance, N. G. Pace and F. B. Jensen, Eds. Dordrecht: Kluwer, 2002.
[3] J. F. Lynch, A. E. Newhall, B. Sperry, G. Gawarkiewicz, A. Fredricks, P. Tyack, C.-S. Chiu, and P. Abbot, "Spatial and temporal variations in acoustic propagation characteristics at the New England shelfbreak front," IEEE J. Oceanic Eng., vol. 28, pp. 129-150, 2003.

[4] M. H. Orr, B. H. Pasewark, S. N. Wolf, J. F. Lynch, T. Schroeder, and C.-S. Chiu, "South China Sea internal tide/internal waves-Impact on the temporal variability of horizontal array gain at $276 \mathrm{~Hz}$,' IEEE J. Oceanic Eng., vol. 29, pp. 1292-1307, Oct. 2004.

[5] P. C. Mignerey and M. H. Orr, "Observations of matched-field autocorrelation time in the South China Sea," IEEE J. Oceanic Eng., vol. 29, pp. 1280-1291, Oct. 2004.

[6] A. Newhall, L. Costello, T. Duda, J. Dunn, G. Gawarkiewicz, J. Irish, J. Kemp, N. McPhee, S. Liberatore, J. Lynch, W. Ostrom, T. Schroeder, R. Trask, and K. von der Heydt, "Preliminary acoustic and oceanographic observations from the ASIAEX 2001 South China Sea experiment," Woods Hole Oceanograph. Inst., Woods Hole, MA, Tech. Rep. WHOI-2001-12, 2001.

[7] S. C. Schock, "Remote estimates of the physical and acoustic properties of sediments in the South China Sea using a chirp sonar and biot theory," IEEE J. Oceanic Eng., vol. 29, pp. 1200-1217, Oct. 2004.

[8] Y.-T. Lin, "personal communication," unpublished, 2003.

[9] G. Gawarkiewicz, J. Wang, M. Caruso, S. Ramp, K. Brink, and F. Bahr, "Shelfbreak circulation and thermohaline structure in the northern South China Sea-contrasting Spring 2000 and Spring 2001," IEEE J. Oceanic Eng., vol. 29, pp. 1131-1143, Oct. 2004.

[10] T. F. Duda and J. C. Preisig, "A modeling study of acoustic propagation through moving shallow-water solitary wave packets," IEEE J. Oceanic Eng., vol. 24, pp. 16-32, 1999.

[11] T. F. Duda, "Acoustic mode coupling by nonlinear internal wave packets in a shelfbreak front area," IEEE J. Oceanic Eng., vol. 29, pp. 118-125, 2004.

[12] C.-S. Chiu, S. R. Ramp, C. W. Miller, J. F. Lynch, T. F. Duda, and T.-Y. Tang, "Acoustic intensity fluctuations induced by south china sea internal tides and solitons," IEEE J. Oceanic Eng., vol. 24, pp. 16-32, 1999.

[13] W. H. Press, S. A. Teukolsky, W. T. Vetterling, and B. P. Flannery, Numerical Recipes in C (Second Edition). New York: Cambridge Univ. Press, 1992.

[14] A. Fredricks, J. A. Colosi, J. F. Lynch, G. Gawarkiewicz, C.-S. Chiu, and P. Abbot, "Analysis of multipath scintillations observed during the summer 1996 New England shelfbreak PRIMER study,” J. Acoust. Soc. Amer., to be published.

[15] T. F. Duda, "Relative influences of various environmental factors on 50-1000 Hz sound propagation in shelf and slope areas," in Impact of Littoral Environmental Variability on Acoustic Predictions and Sonar Performance, N. G. Pace and F. B. Jensen, Eds. Dordrecht: Kluwer, 2002.

[16] I. Dyer, "Statistics of sound propagation in the ocean," J. Acoust. Soc. Amer., vol. 48, pp. 337-345, 1970.

[17] R. H. Headrick, J. F. Lynch, J. N. Kemp, K. von der Heydt, J. Apel, M. Badiey, C.-S. Chiu, S. Finette, M. Orr, B. Pasewark, A. Turgut, S. Wolf, and D. Tielbuerger, "Acoustic normal mode fluctuation statistics in the 1995 SWARM internal wave scattering experiment," J. Acoust. Soc. Amer, vol. 107, pp. 201-220, 2000.

[18] J. M. Martin and S. M. Flatte', "Simulation of point-source scintillation through three-dimensional random media," J. Opt. Soc. Amer. A, vol. 7, pp. 838-847, 1990.

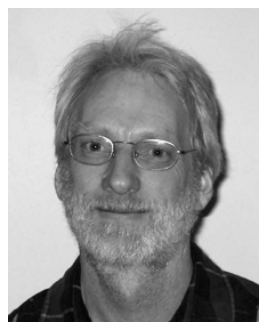

Timothy F. Duda received the B.A. degree in physics from Pomona College, Claremont, CA, in 1979 and the Ph.D. degree in oceanography from the Scripps Institution of Oceanography, University of California, San Diego, in 1986.

He has been a Scientist with the Woods Hole Oceanographic Institution (WHOI), Woods Hole, MA, since 1991. Prior to that, he held positions with the University of California, Santa Cruz. His three primary fields of study are ocean acoustic propagation, ocean internal gravity waves, and ocean mixing processes. He has modeled and analyzed fluctuations of ocean acoustic transmissions, has made electromagnetic-type internal-wave measurements, and performed in situ measurements of ocean microstructure.

Dr. Duda is a Member of the American Meteorological Society, the American Geophysical Union, and the Acoustical Society of America. 


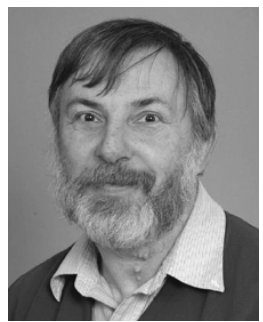

James F. Lynch (M'96-SM'03) was born in Jersey City, NJ, on June 3, 1950. He received the B.S. degree in physics from Stevens Institute of Technology, Hoboken, NJ, in 1972 and the Ph.D. degree in physics from the University of Texas, Austin, in 1978.

He was with the Applied Research Laboratories, University of Texas at Austin (ARL/UT) from 1978 to 1981 , after which he joined the scientific staff at the Woods Hole Oceanographic Institution (WHOI), Woods Hole, MA. He has been with WHOI since then and currently holds the position of Senior Scientist in the Applied Ocean Physics and Engineering Department. His research specialty areas are ocean acoustics and acoustical oceanography, but he also greatly enjoys occasional forays into physical oceanography, marine geology, and marine biology.

Dr. Lynch is a Fellow of the Acoustical Society of America and Editor-inChief of the IEEE JOURNAL OF OCEANIC ENGINEERING.

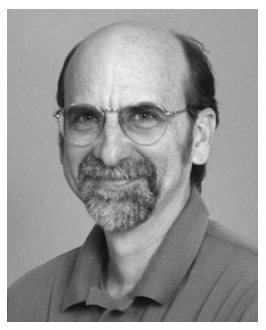

Arthur E. Newhall received the B.S. degree in mathematics from the University of Maine, Orono, in 1985.

$\mathrm{He}$ is a Research Specialist with the Applied Ocean Physics and Engineering Department, Woods Hole Oceanographic Institution, Woods Hole, MA. His current interests include ocean acoustic propagation modeling, acoustical oceanography, ocean acoustic tomography, and software engineering.

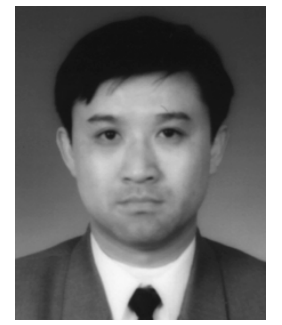

Lixin Wu received the B.Sc. degree in physics and the M.Sc. degree in underwater acoustics in 1990 and 1993, respectively, from Qingdao Ocean University, Qingdao, China, and the Ph.D. degree, also in underwater acoustics, from the Institute of Acoustics, Chinese Academy of Sciences, Beijing, in 2001.

$\mathrm{He}$ has been a Associate Researcher with the Institute of Acoustics since 2001. Prior to that, he held positions with Qingdao Ocean University. His three primary fields of study are ocean acoustic propagation, ocean acoustic inversion, and underwater acoustic signal processing. He has modeled and analyzed fluctuations of ocean acoustic transmissions, has performed inversions for acoustical parameters of the sea bottom, and performed long-range underwater acoustic communication based on Chaotic-FM M-ary.

Dr. Wu is a Member of the Chinese Oceanography Society and the Acoustical Society of China.

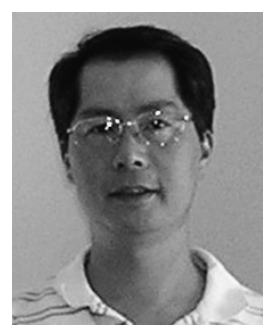

Ching-Sang Chiu received the Ph.D. degree from the Massachusetts Institute of Technology/Woods Hole Oceanographic Institution, Woods Hole, MA (MIT-WHOI) joint program in 1985.

$\mathrm{He}$ is a Professor of oceanography with the Naval Postgraduate School. His research specialties include ocean acoustics, acoustical oceanography, and coastal ocean processes and their influences on acoustics prediction. He has authored or coauthored more than 40 refereed publications in those subject areas.

Dr. Chiu is a Fellow of the Acoustical Society of America and Editor-in-Chief of the Journal of Computational Acoustics. 